\title{
Microstructure evolution during recrystallization of dual-phase steels
}

D. Raabe, F. Roters, D. Ponge, S. Zaefferer, N. Perannio, M. Calcagnotto

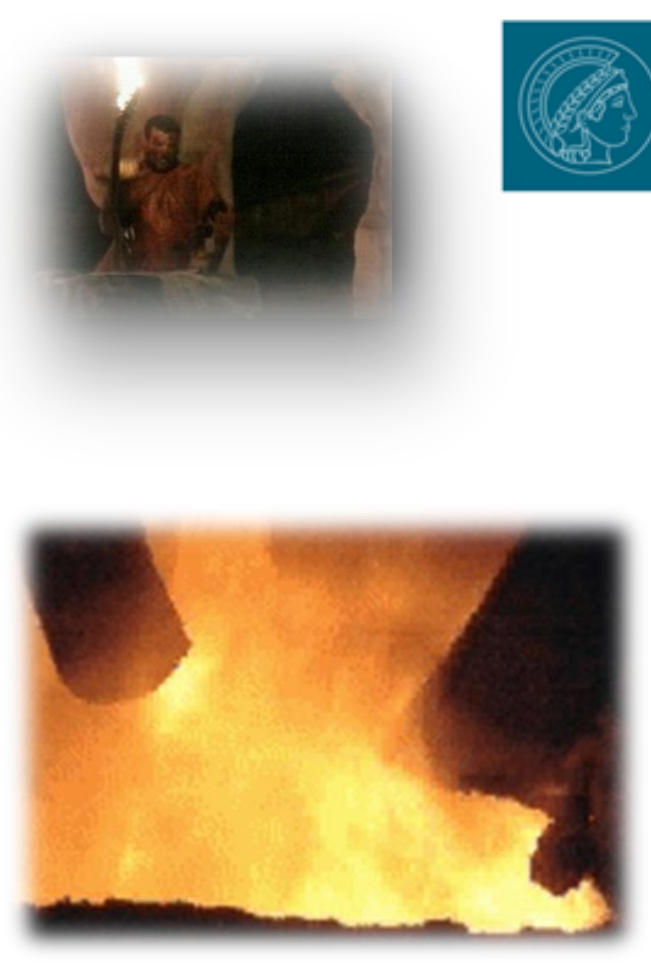

Max-Planck-Institut für Eisenforschung $\mathrm{GmbH}$ Düsseldorf, Germany

WWW.MPIE.DE d.raabe@mpie.de
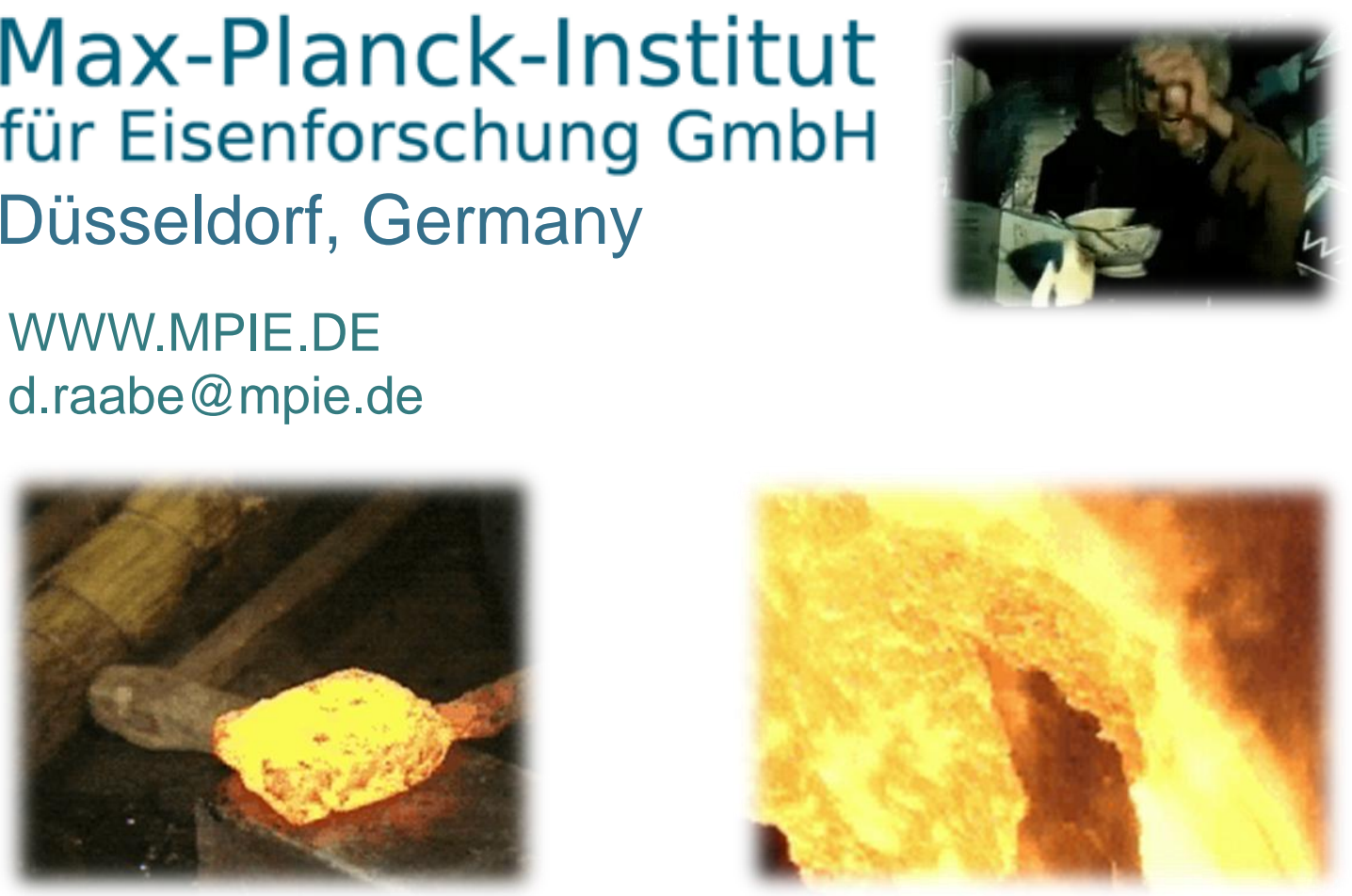

RX\&GG 4, July 2010, Sheffield 
- Motivation

- Experiments

- Microstructure and texture evolution

- 3D tomographic analysis of interface regions

- Correlation to DP mechanical properties

- Ultra-fine grained DP

- Conclusions 
- Low energy consumption through weight reduction: high-strength steels with a good formability such as C Mn DP

- Microstructure evolution in hot rolled, cold rolled, and hot-dip galvanized dual-phase steel sheets

- Dominating mechanisms during intercritical annealing: recovery, recrystallization, phase transformation and competition between them

- Important role of annealing parameters such as heating rate, intercritical annealing temperature, annealing time, cooling rate, and the final annealing temperature

- Through-thickness inhomogeneity: introduced by hot and cold rolling, leading to a planestrain texture in the center layer and a shear texture close to the surface of the sheets

- Microstructure and texture evolution in DP steels considering through-thickness inhomogeneity

- Spatial distribution of the DP constituents through-thickness

- Annealing experiments in a wide temperature and time range
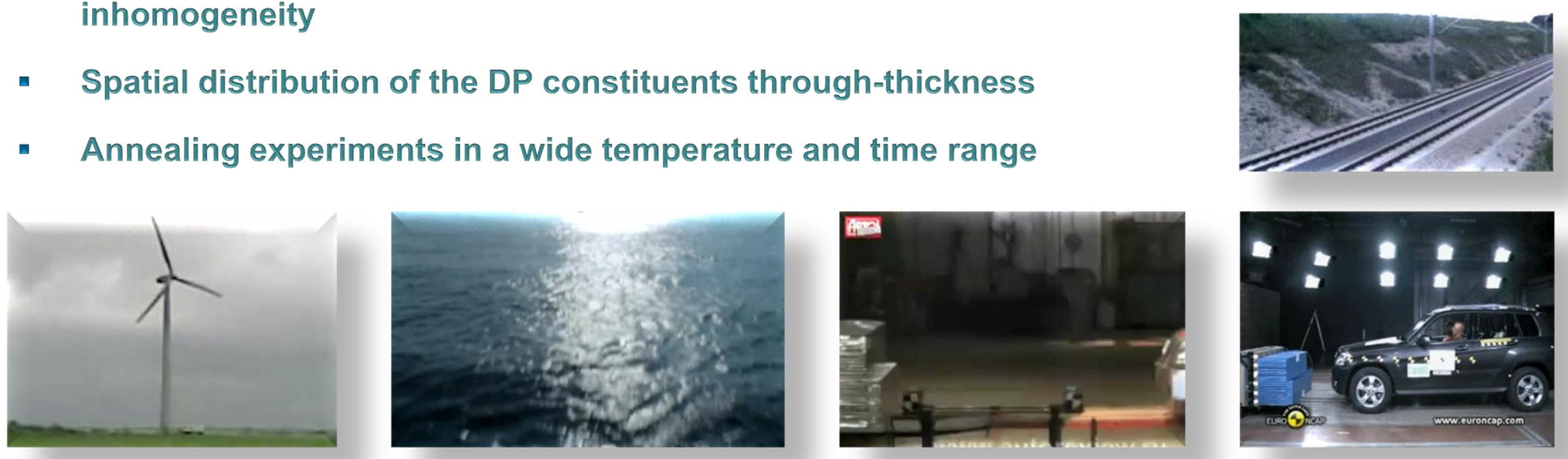
- Motivation

- Experiments

- Microstructure and texture evolution

- 3D tomographic analysis of interface regions

- Correlation to DP mechanical properties

- Ultra-fine grained DP

- Conclusions 
Chemical composition of hot band in wt. \%.

\begin{tabular}{ccccccccc}
\hline \hline Fe & C & Si & Mn & P & S & N & Al & Cu \\
bal. & 0.147 & 0.403 & 1.868 & 0.01 & 0.002 & 0.0056 & 0.037 & 0.028 \\
\hline Cr & Ni & V & Ti & Nb & Mo & Sn & B & \\
0.028 & 0.044 & 0.098 & 0.005 & 0.047 & 0.003 & 0.009 & 0.0001 & \\
\hline \hline
\end{tabular}

Hot rolled to $3.75 \mathrm{~mm}$ as starting material

Volume fractions of $65 \%$ ferrite and $35 \%$ pearlite

Cold rolled to thicknesses of $2.2 \mathrm{~mm}, 1.75 \mathrm{~mm}$, and $1.5 \mathrm{~mm}$ 
- hot rolled (3.5 mm, 70\% ferrite, 30\% pearlite, 0.147 wt $\%$ C, 1.9 wt. \% $\mathrm{Mn}, 0.4$ wt.\% Al)

- cold rolled in laboratory $(1.70 \mathrm{~mm}, 50 \%)$

- industrially cold rolled (2.2 mm, $1.75 \mathrm{~mm}, 1.5 \mathrm{~mm}, 63 \%, 50 \%, 43 \%)$

- annealing of industrially cold rolled $(1.75 \mathrm{~mm}, 50 \%)$ sheets

* salt bath (MPIE) / conductive annealing (SZMF)

annealing temperature $740^{\circ} \mathrm{C} \approx \mathrm{Ac}_{1}, 860^{\circ} \mathrm{C} \approx \mathrm{Ac}_{3}$, and $920^{\circ} \mathrm{C}$

* annealing time $100 \mathrm{~s}, 200 \mathrm{~s}$, and $300 \mathrm{~s}$

* cooling rate $7 \mathrm{~K} / \mathrm{s}, 15 \mathrm{~K} / \mathrm{s}$, and $22 \mathrm{~K} / \mathrm{s}$

* heating rate $10 \mathrm{~K} / \mathrm{s}, 20 \mathrm{~K} / \mathrm{s}$, and $30 \mathrm{~K} / \mathrm{s}$ 


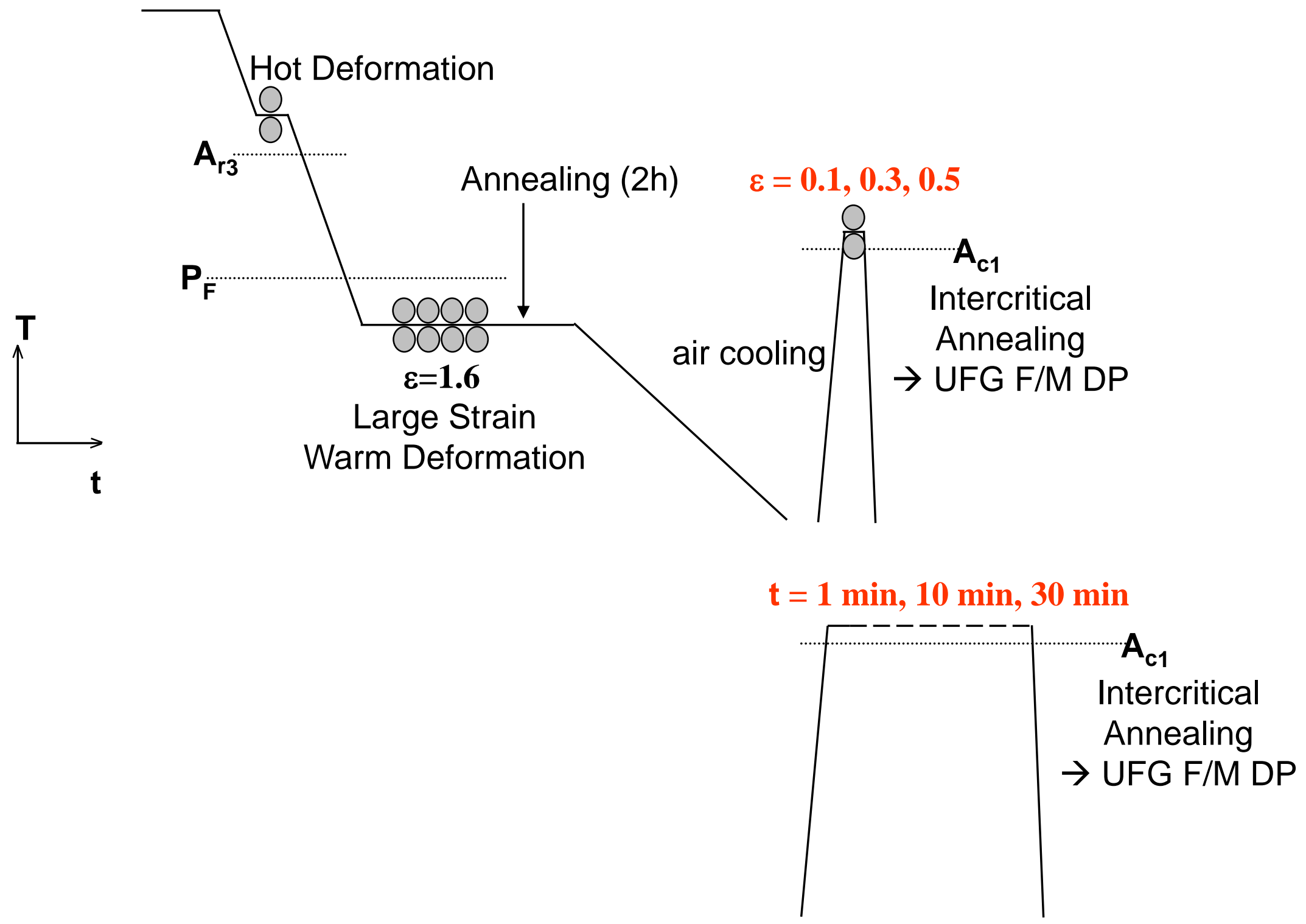




\section{recrystallization dual phase steels}

phase changes in dual phase steels

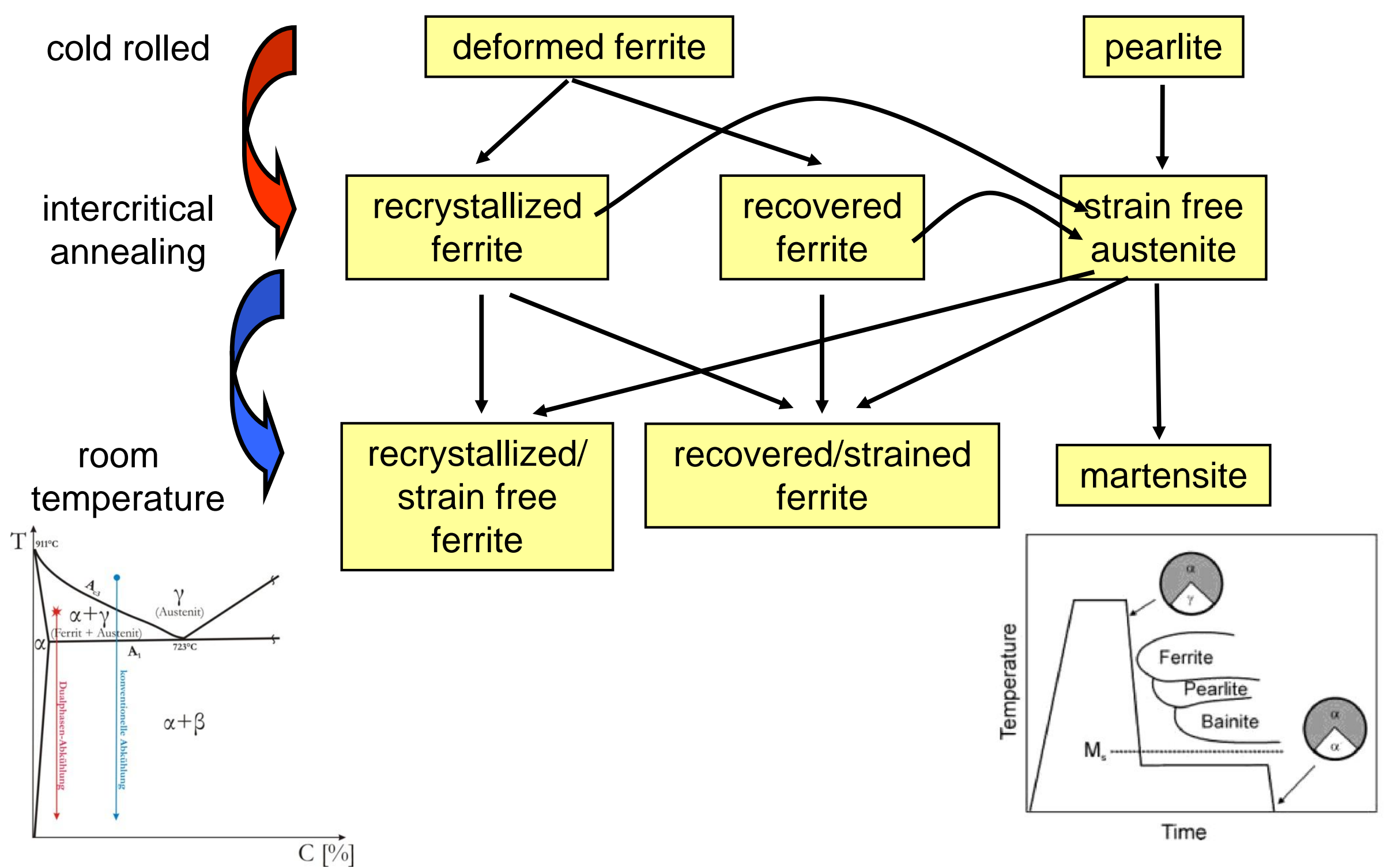


- Motivation

- Experiments

- Microstructure and texture evolution

- 3D tomographic analysis of interface regions

- Correlation to DP mechanical properties

- Ultra-fine grained DP

- Conclusions 


\section{Microstructure evolution}
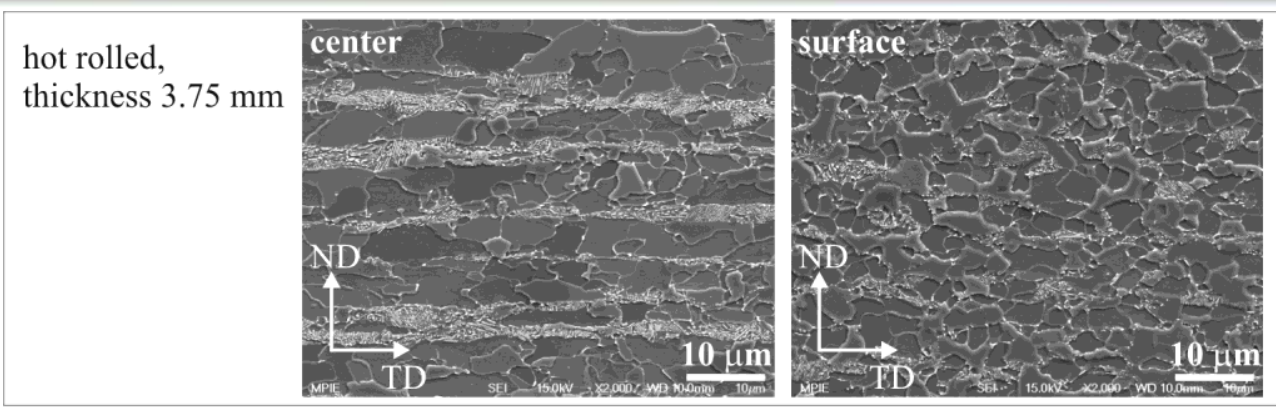

cold rolled, thickness $1.75 \mathrm{~mm}$
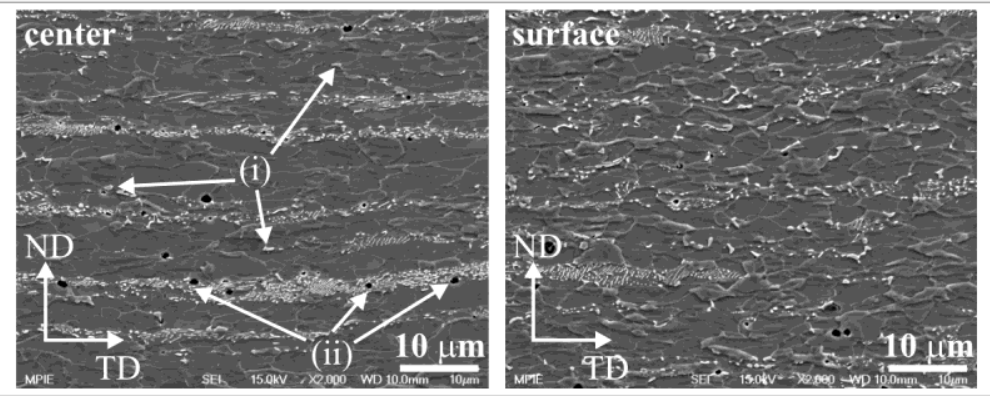

hot-dip galvanized at $830^{\circ} \mathrm{C}$ for $80 \mathrm{~s}$
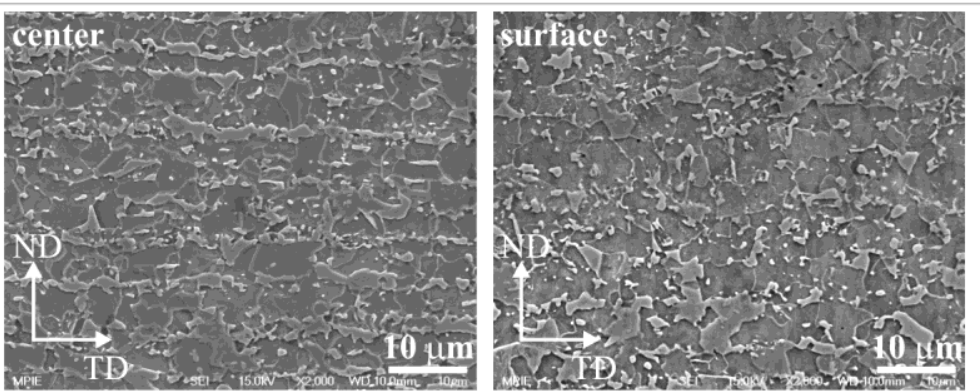

conductively
annealed at
$920^{\circ} \mathrm{C}$ for $100 \mathrm{~s}$,
heating rate $20 \mathrm{~K} / \mathrm{s}$,
cooling rate $-15 \mathrm{~K} / \mathrm{s}$
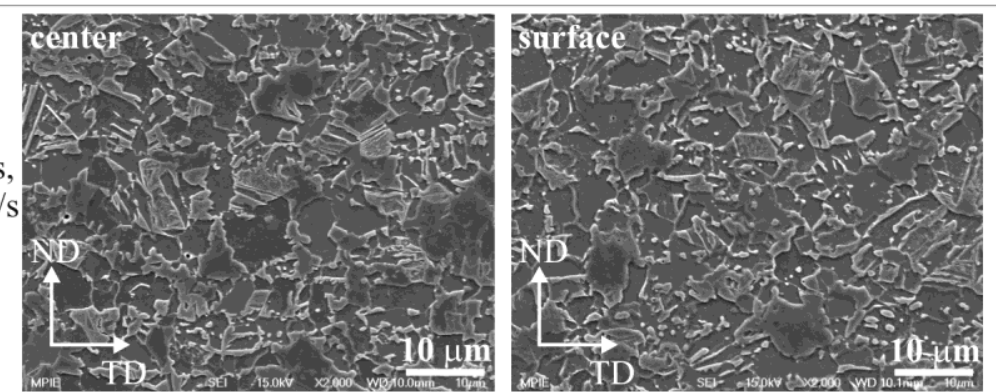

\section{Max-Planck-Institut für Eisenforschung, Düsseldorf, Germany}




\section{Microstructure and texture evolution - hot band through thickness}

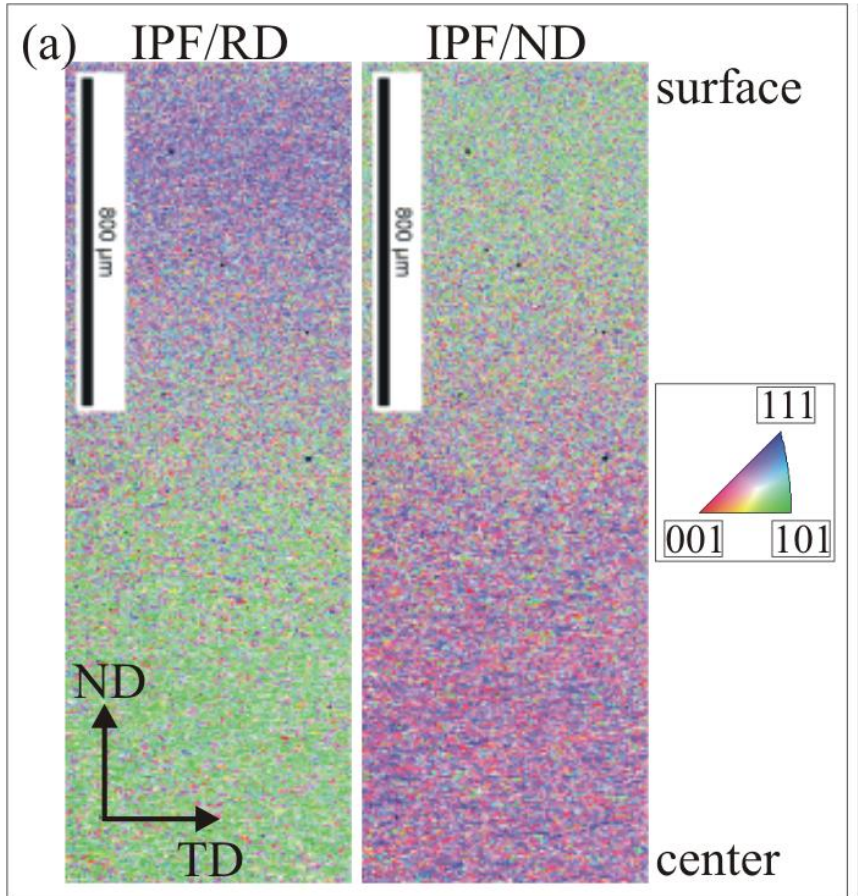

(c) $\varphi_{22}=45^{\circ}, \mathrm{s}=0.013$, center $\varphi_{2_{2}}=0^{\circ}, \mathrm{s}=0.013$,

$$
\begin{array}{ccc}
f(g)_{\max }=11.9 & \mathrm{~s} \approx 0 & f(g)_{\max }=6.91 \\
\varphi_{1}\left({ }^{\circ}\right) & & \varphi_{1}\left(^{\circ}\right)
\end{array}
$$

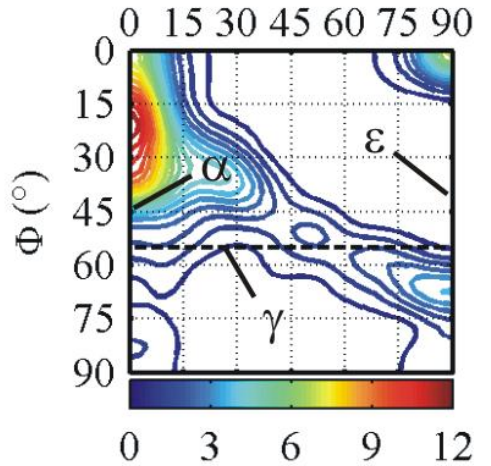

(b)

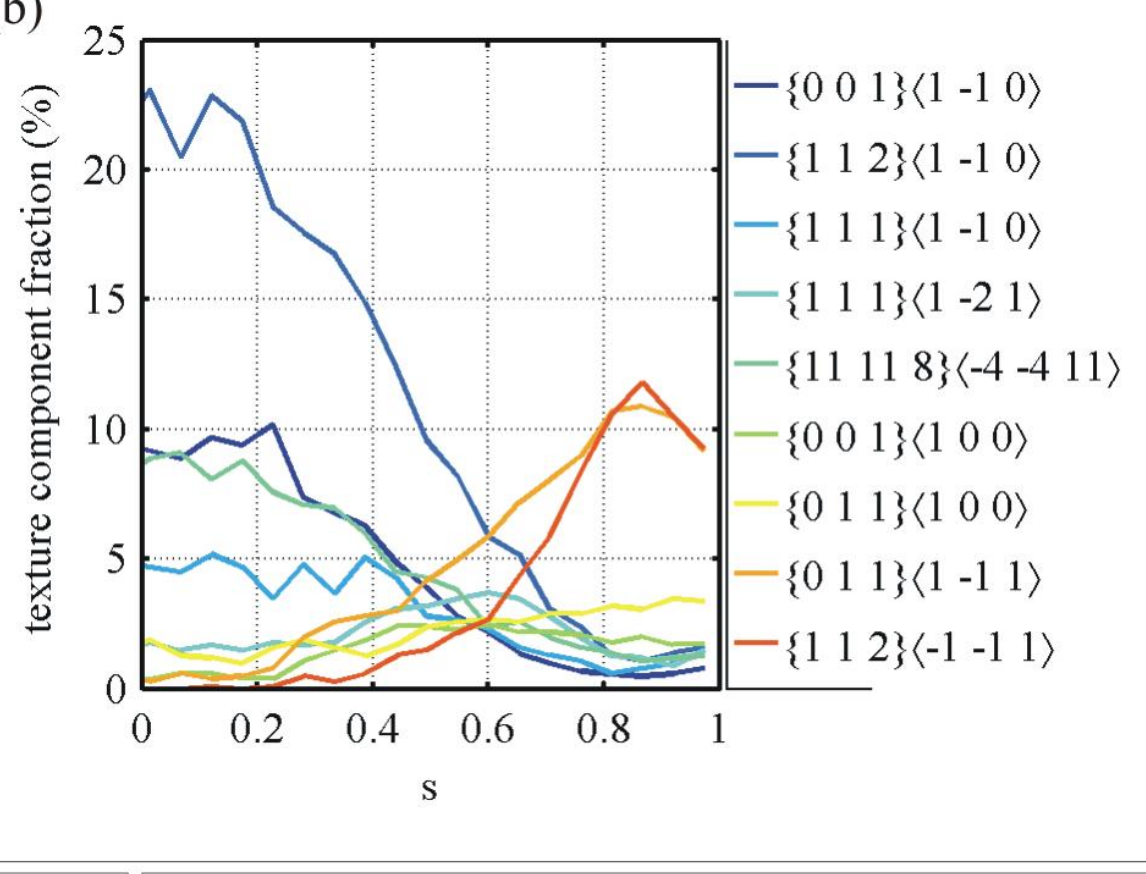

(d) $\varphi_{2}=45^{\circ}, \mathrm{s}=0.87$, surface $\varphi_{22}=0^{\circ}, \mathrm{s}=0.87$,

$$
\begin{array}{ccc}
f(g)_{\max }=3.77 & \mathrm{~s} \approx 0.9 & f(g)_{\max }=3.77 \\
\varphi_{1}\left(^{\circ}\right) & \left.\varphi_{1}{ }^{\circ}{ }^{\circ}\right)
\end{array}
$$
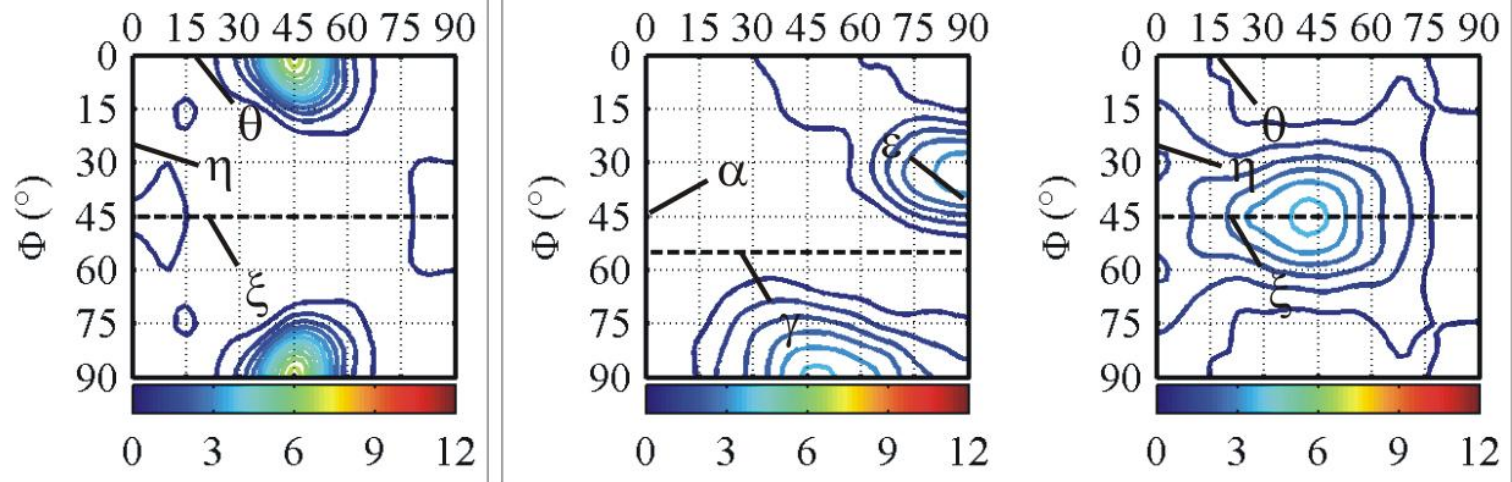

Max-Planck-Institut für Eisenforschung, Düsseldorf, Germany 


\section{Texture evolution -rolled, annealed, through thickness}
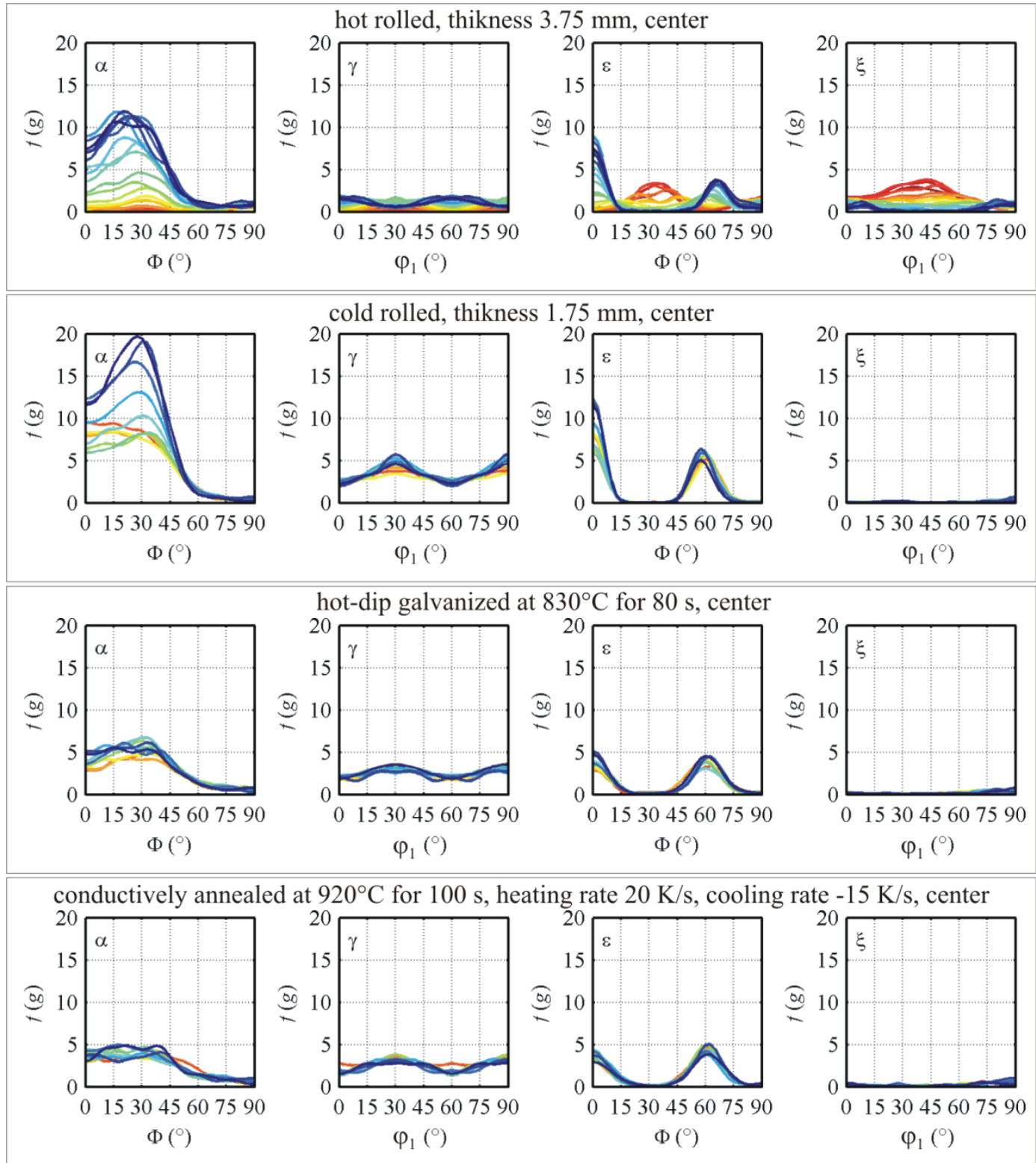

\section{Max-Planck-Institut für Eisenforschung, Düsseldorf, Germany}




\section{Results - EBSD - cold rolled}
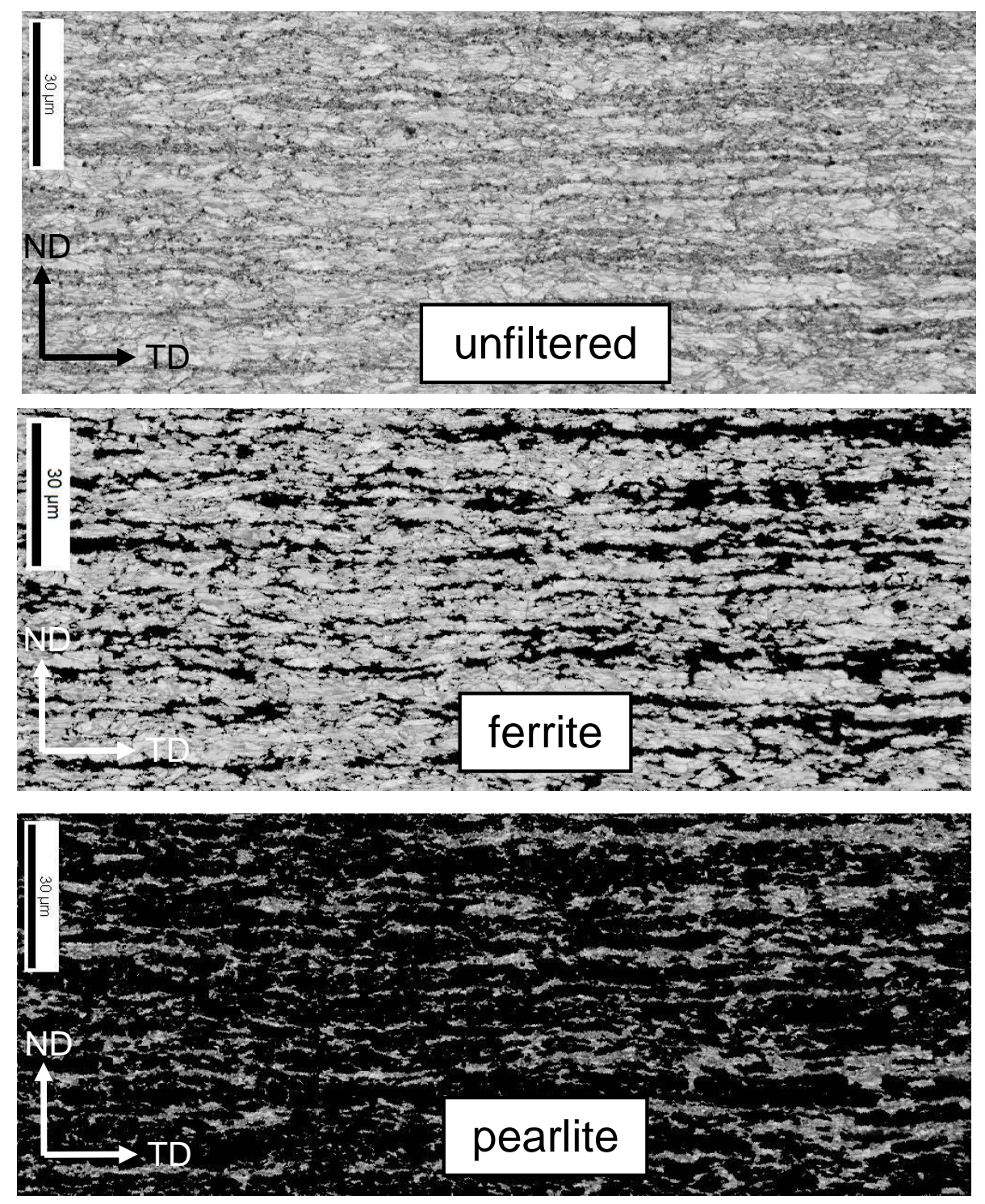

cold rolled, center of sample

image quality signal allows separate analysis of the constituents

$>$ ferrite volume fraction $74 \%$ grain size $4.8 \mu \mathrm{m}$, aspect ratio 0.26 $>$ large grains are deformed

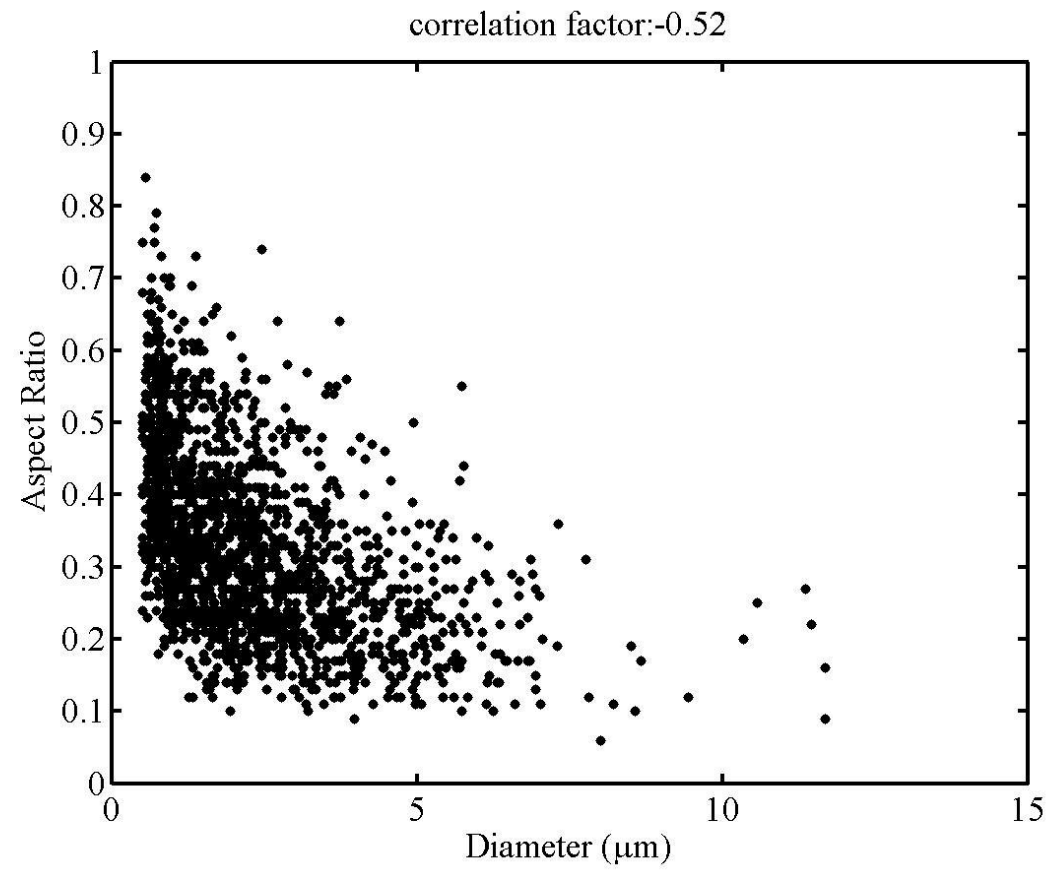




\section{Results - EBSD - cold rolled}

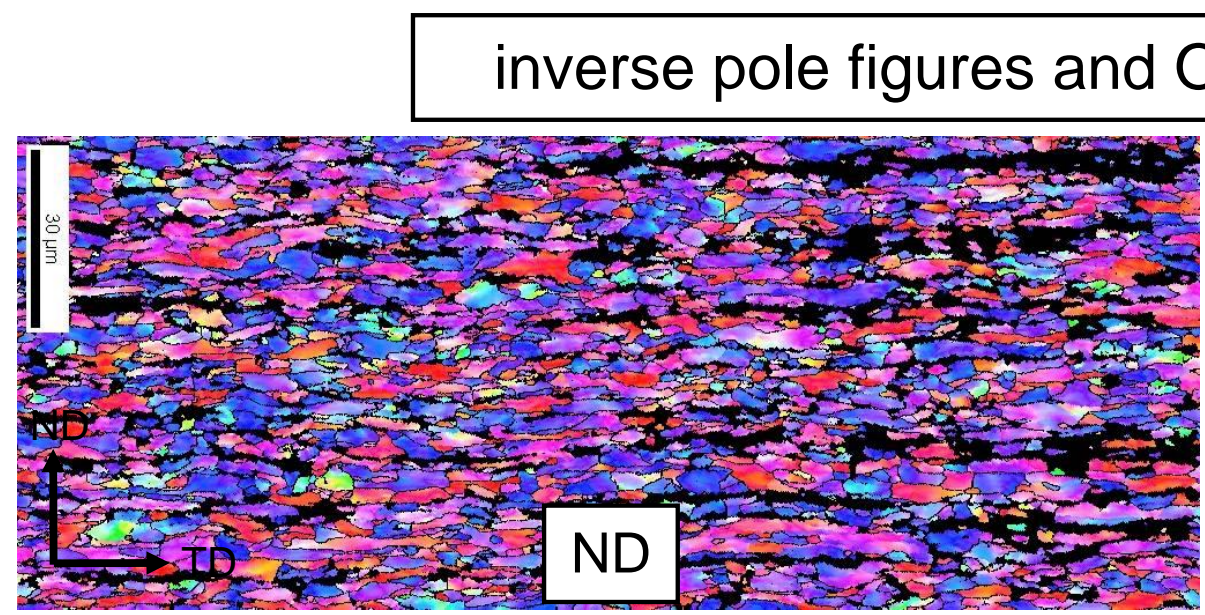

ODF, cold rolled, center

$><110>$ parallel RD ( $\alpha$-fiber $),\{111\}$ parallel ND $(\gamma$-fiber $)$

typical texture for bcc-materials

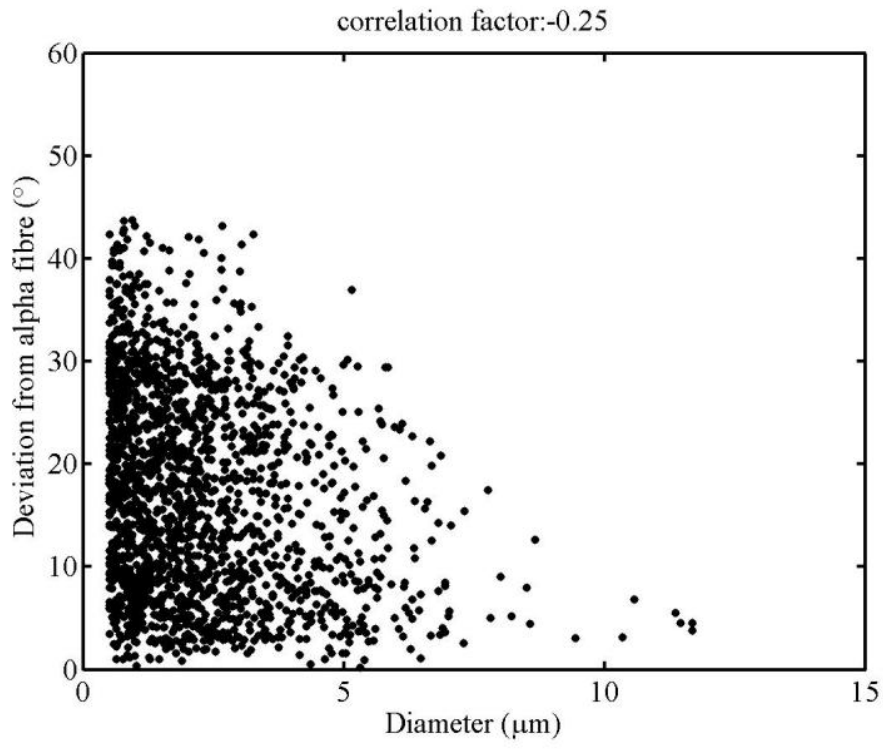

large grains are closer to the $\alpha$-fiber
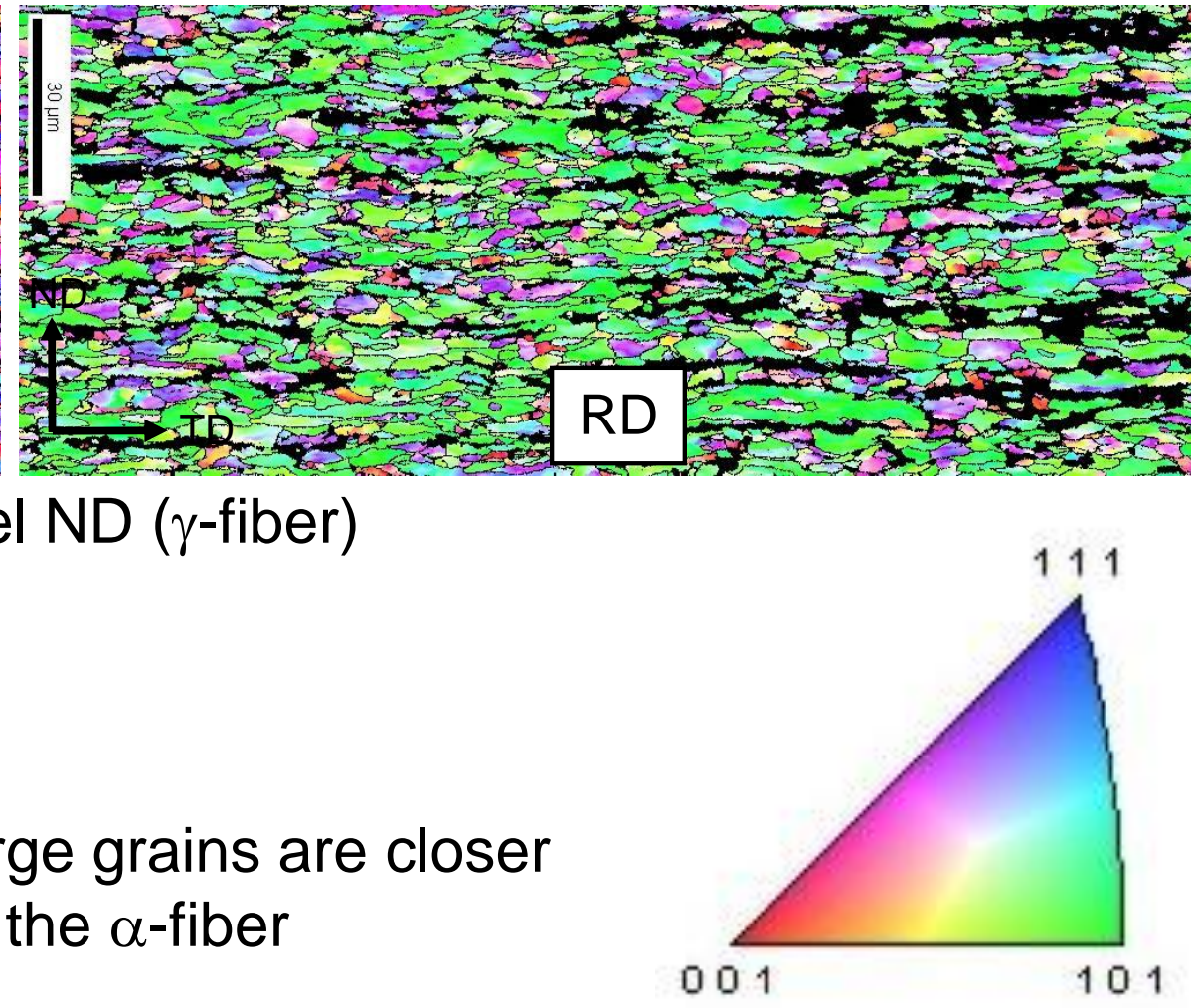
Competition: recrystallization -transformation, through thickness

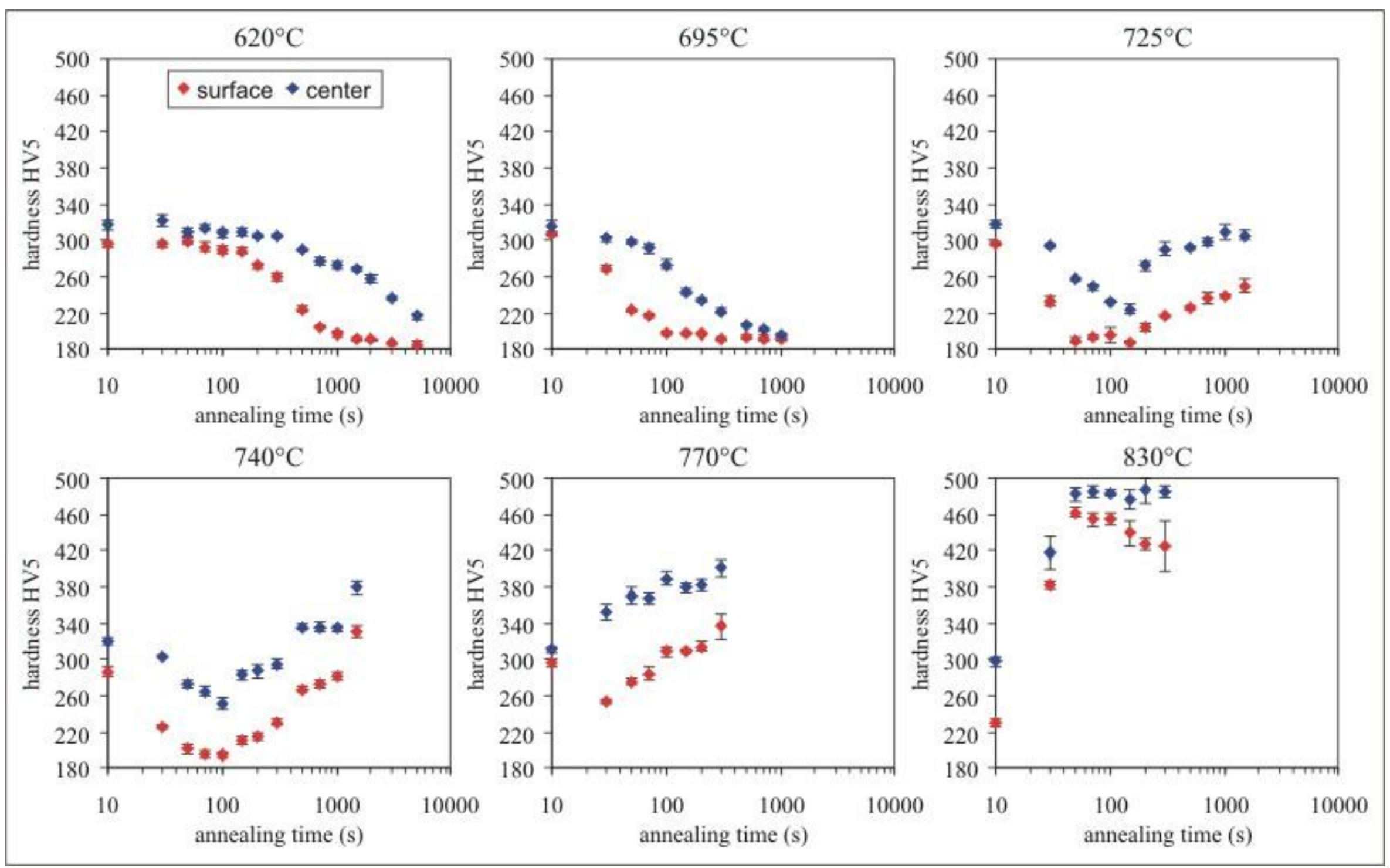

\section{Max-Planck-Institut für Eisenforschung, Düsseldorf, Germany}


annealed in salt bath at $740^{\circ} \mathrm{C}$ for $100 \mathrm{~s}$
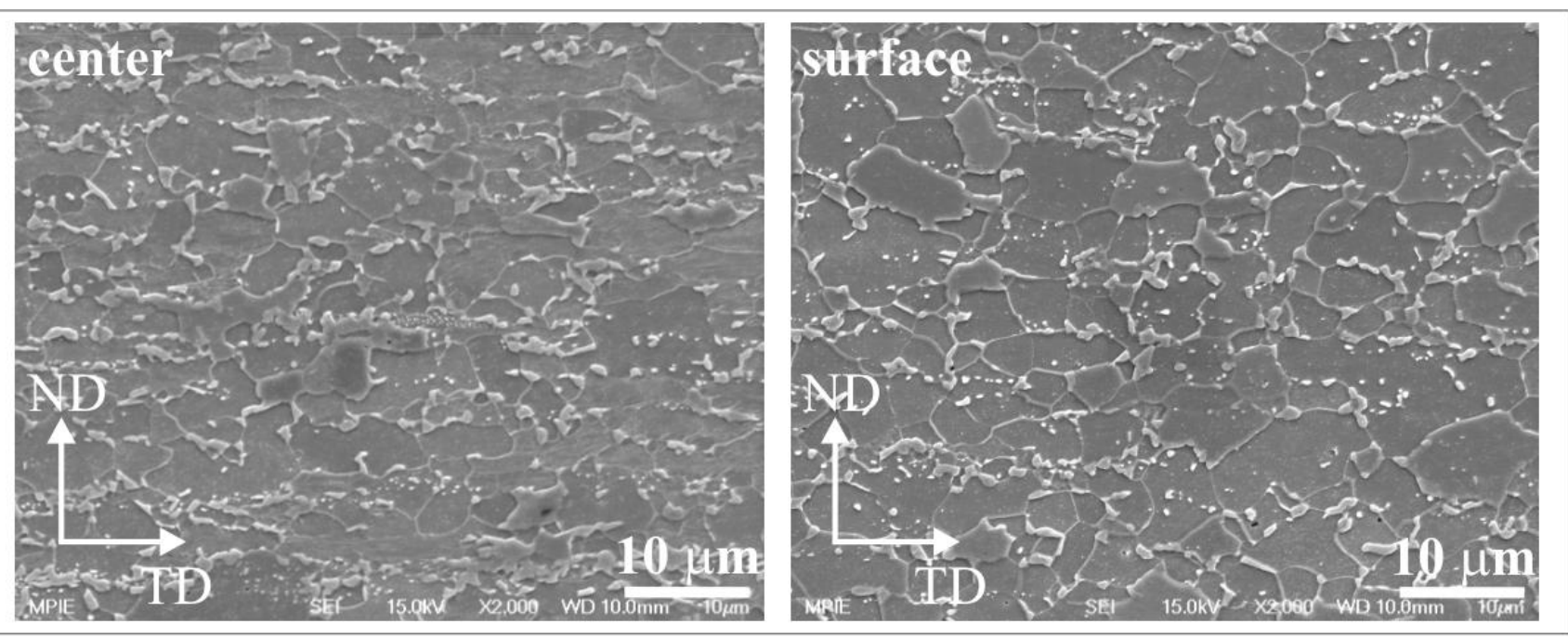

conductively annealed at $740^{\circ} \mathrm{C}$ for $100 \mathrm{~s}$, heating rate $20 \mathrm{~K} / \mathrm{s}$, cooling rate $-15 \mathrm{~K} / \mathrm{s}$
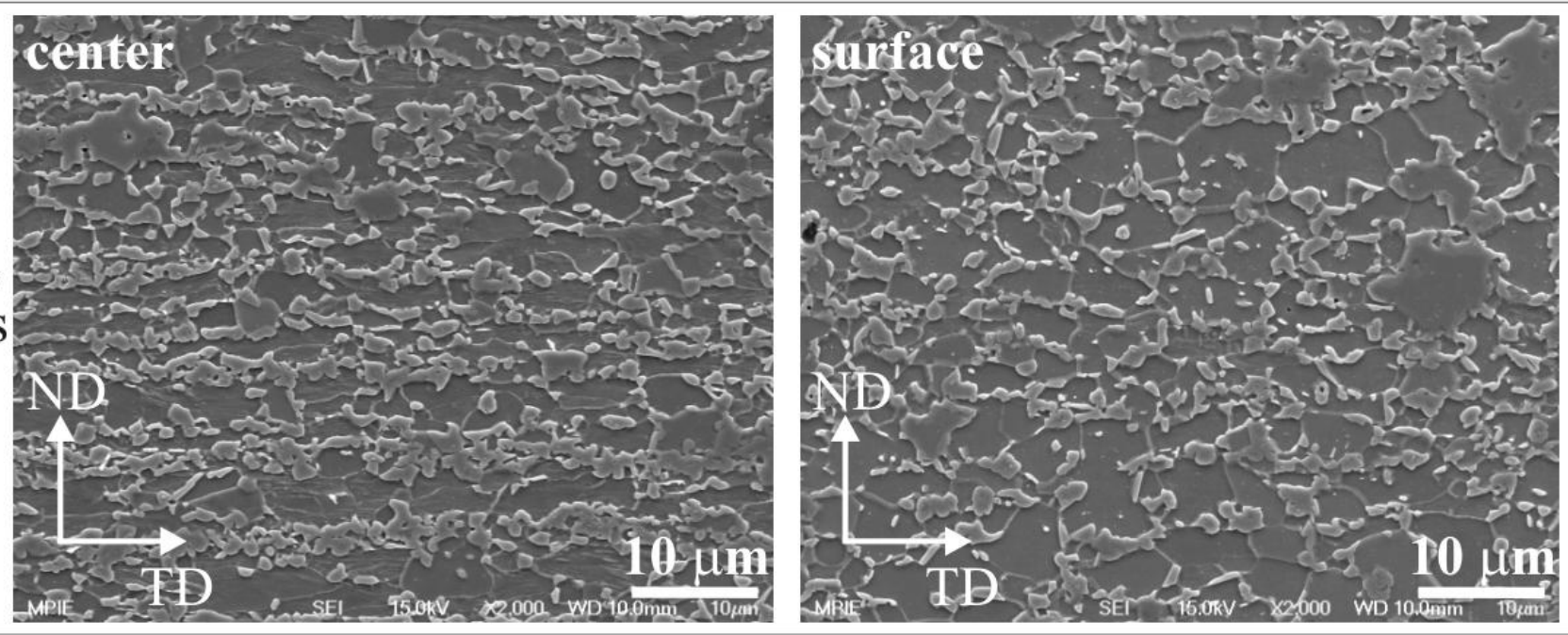


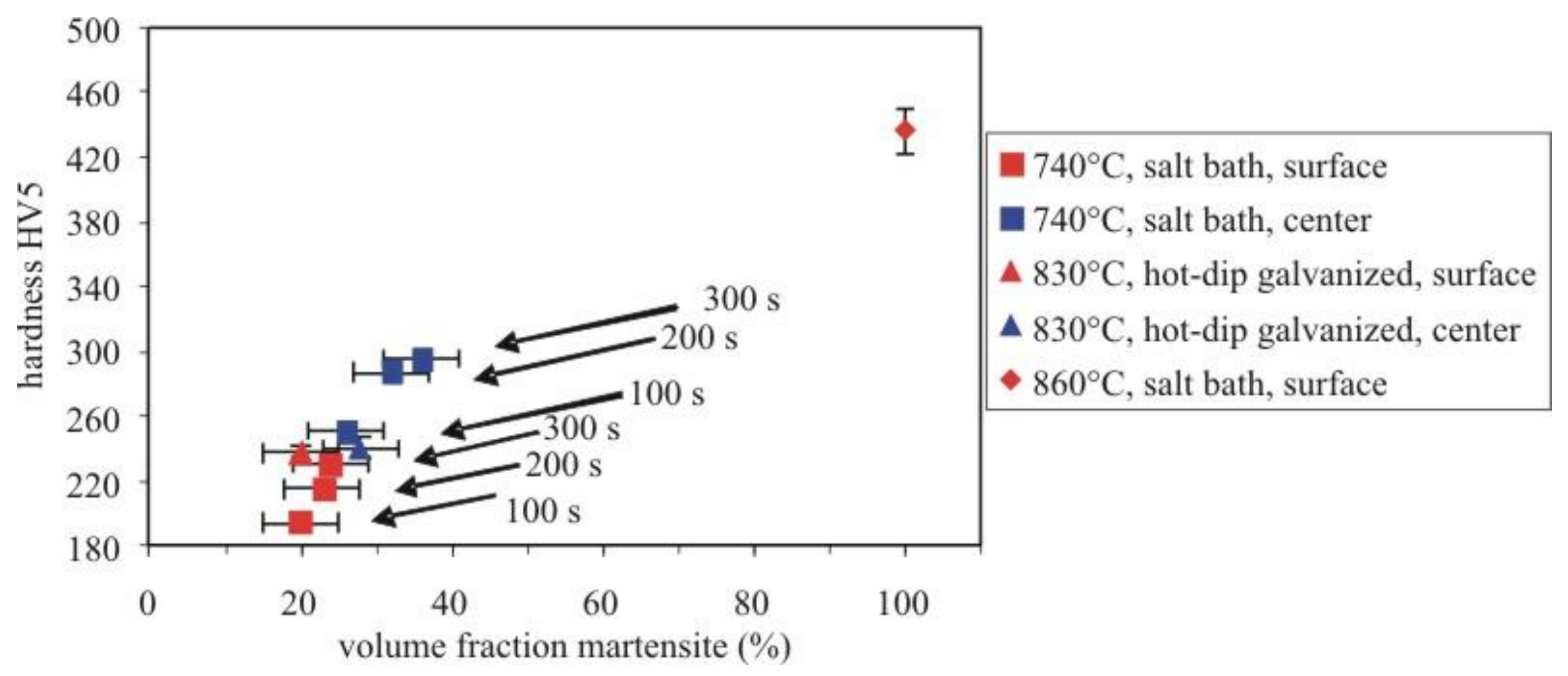



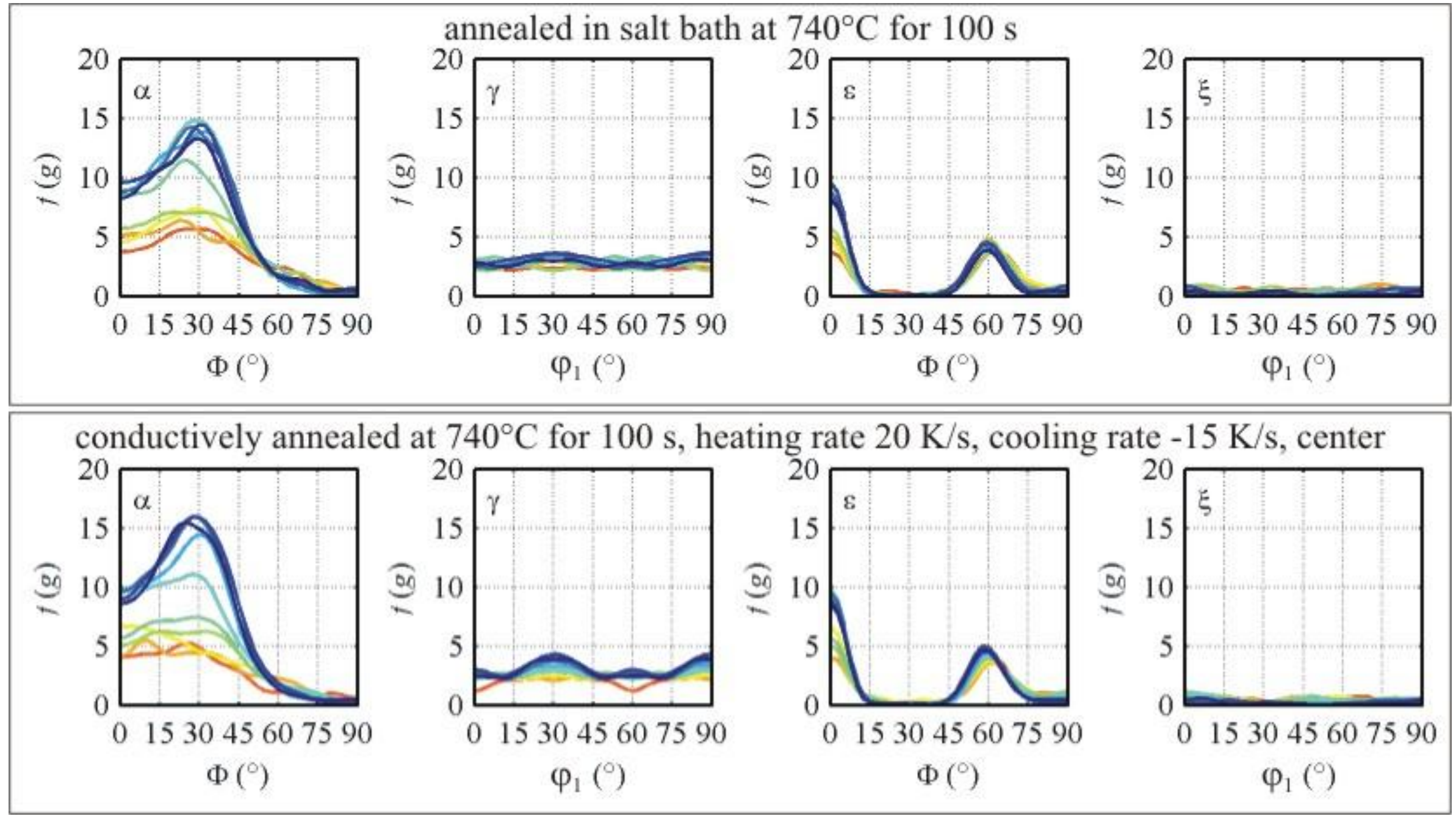

\section{Max-Planck-Institut für Eisenforschung, Düsseldorf, Germany}


- Motivation

- Experiments

- Microstructure and texture evolution

- 3D tomographic analysis of interface regions

- Correlation to DP mechanical properties

- Ultra-fine grained DP

- Conclusions 
- Increase phase space of microstructure analysis $6 \mathrm{D}\left(\varphi_{1}, \phi, \varphi_{2}, \mathrm{x}, \mathrm{y}, \mathrm{z}\right)$ : Crystallography and texture with morphology 8D $\left(\varphi_{1}, \phi, \varphi_{2}, \mathrm{~h}, \mathrm{k}, \mathrm{x}, \mathrm{y}, \mathrm{z}\right)$ : Interface crystallography (interface texture)

- Spatial texture and phases (connectivity, percolation, correlations)

- Sectioning by FIB accurate depth definition, flat parallel sections, high resolution $(<50 \mathrm{~nm})$

- Observation by EBSD good contrast on crystalline material, reconstruction in 2D and 3D, quantitative, high resolution ( $50 \mathrm{~nm}$ ),

- Combination of FIB and EBSD high measurement speed, fully automatic
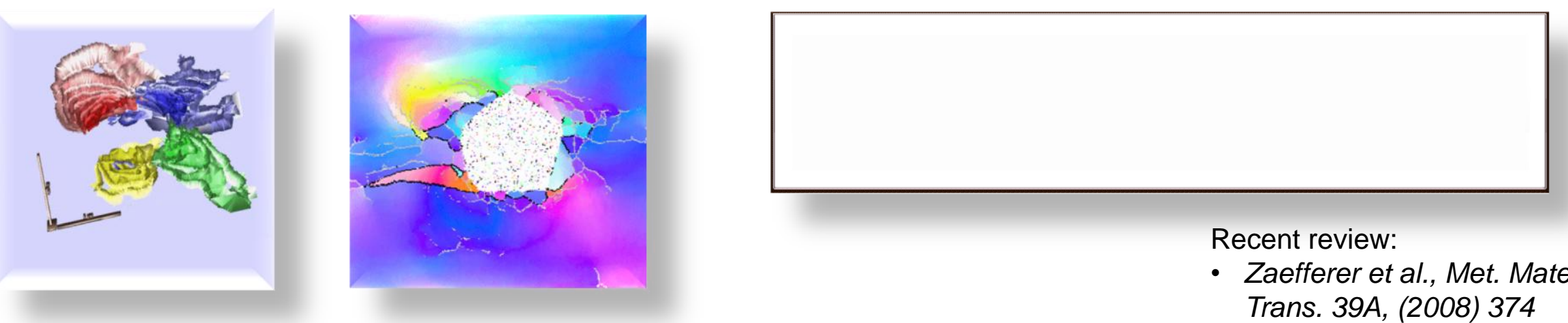

Recent review:

- Zaefferer et al., Met. Mater. Trans. 39A, (2008) 374 


\section{Instrument overview}

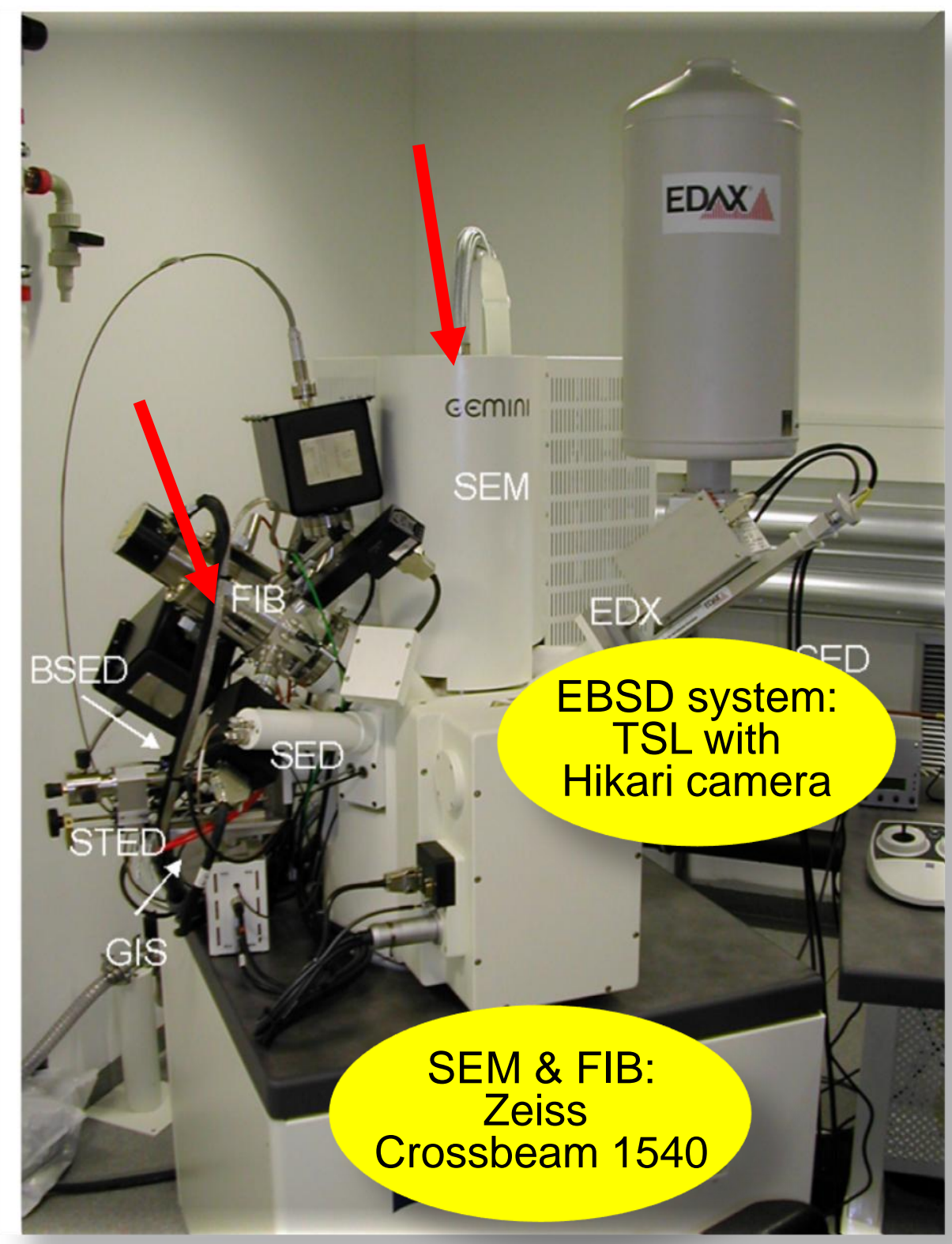

- Scanning electron microscope (SEM)

- observation of microstructure

- Scanning $\mathrm{Ga}^{+}$-ion microscope

(FIB = focused ion beam)

- sputtering of material for serial sectioning

- Quantitative images with EBSD and EDX

- quantitative characterisation of microstructure

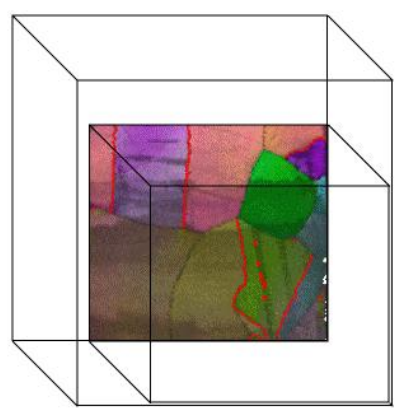



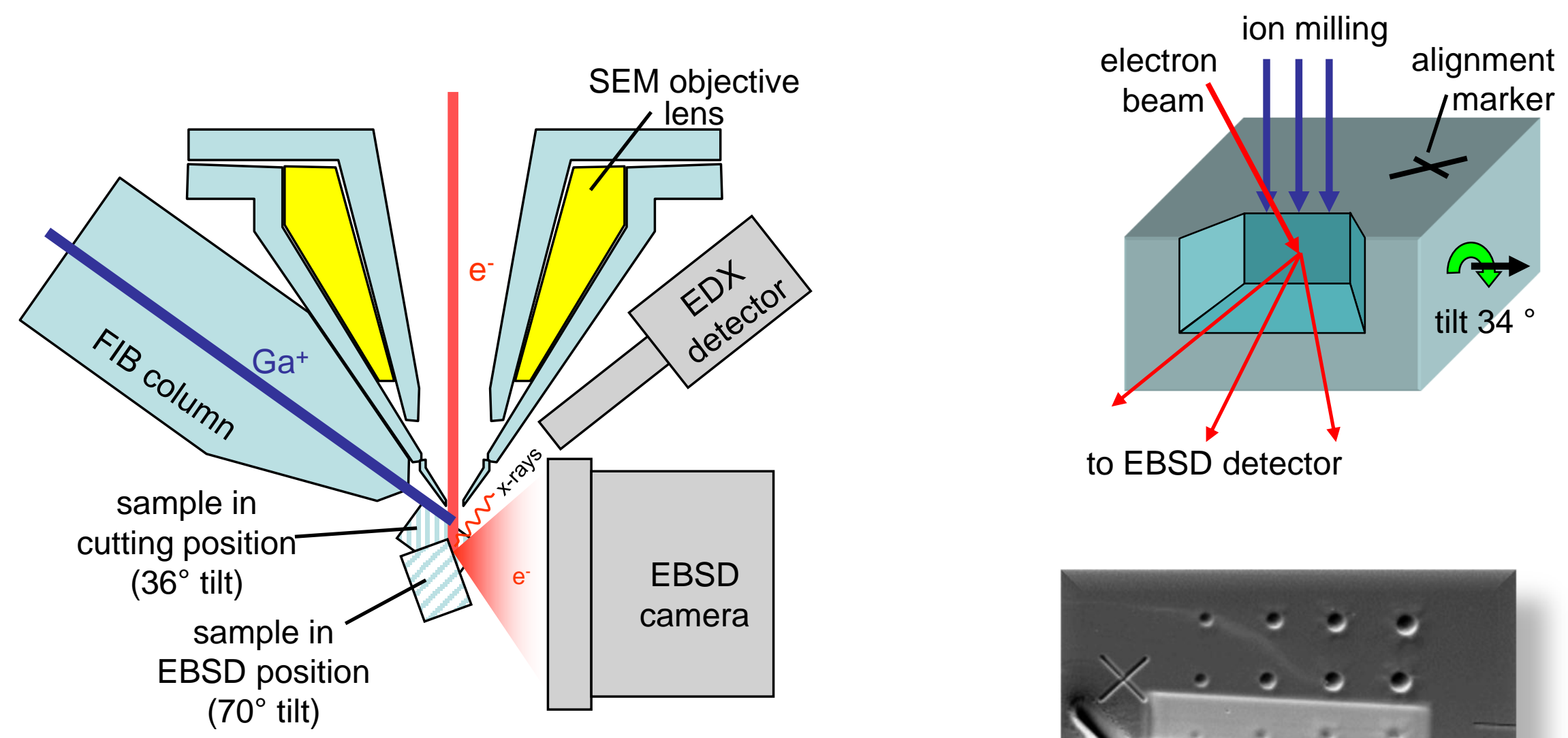

to EBSD detector

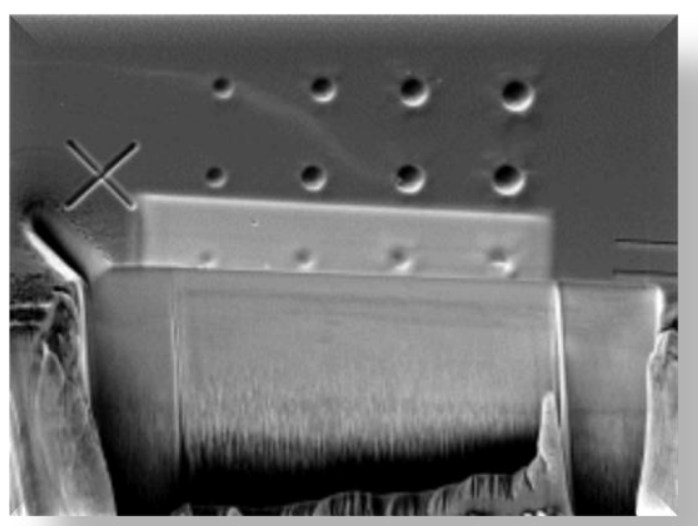




\section{Approaches to 8D interface analysis and 3D GND analysis}

Plane boundaries defined by triple junctions in two adjacent layers

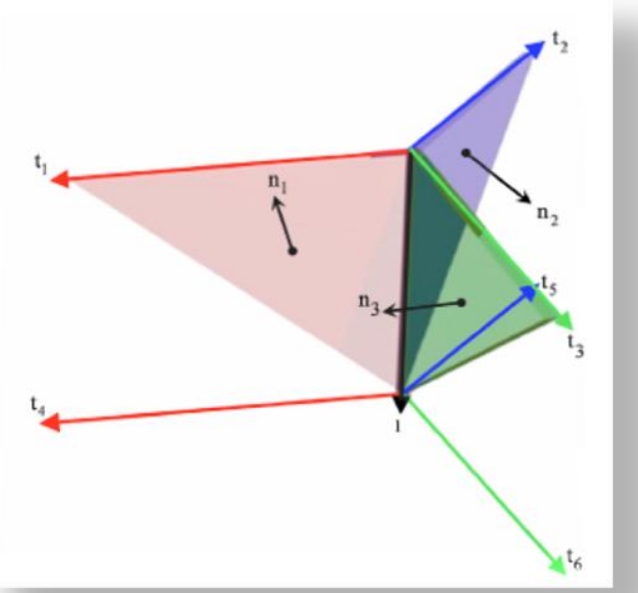

Triangulation the interfaces for defining the grain boundaries

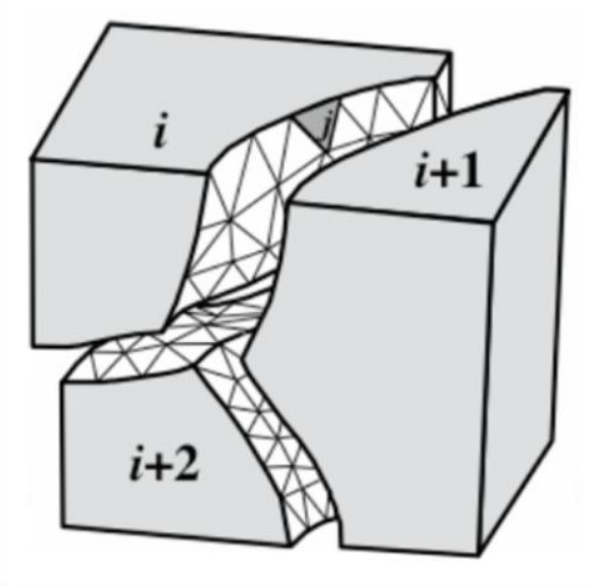

Tony Rollett

GND (Kröner-Nye)
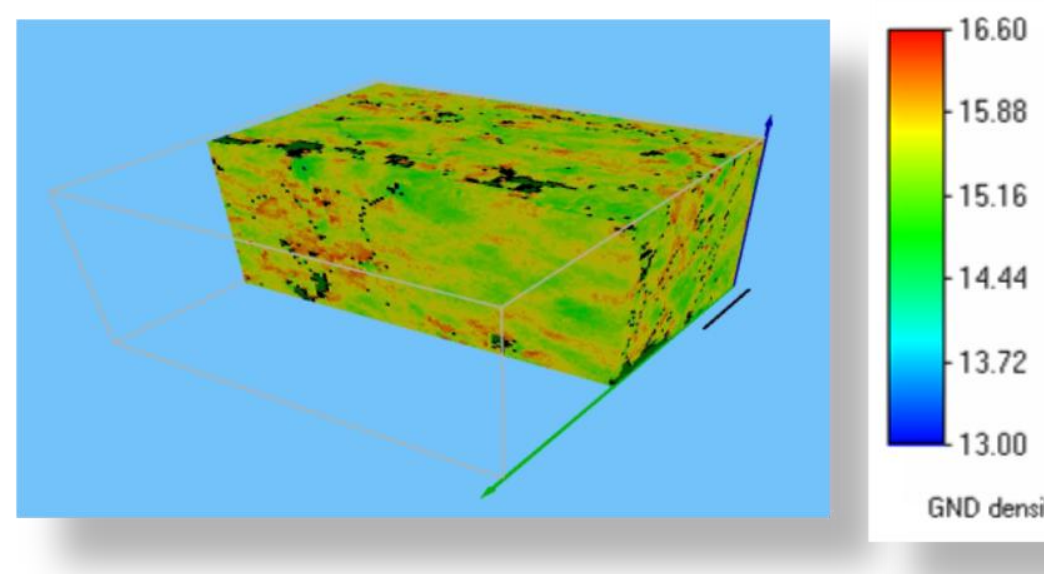

GND density $\log \left[1 / \mathrm{m}^{2}\right]$ 


\section{From local misorientations to GNDs}

$$
\begin{array}{cl}
\triangle \phi=\phi_{(2)} \phi_{(1)}^{-1} & \text { misorientation } \\
|\triangle \phi|=\min \left\{\cos ^{-1}\left\{\operatorname{tr}\left[\left(O_{i}^{c r y} \phi_{(1)}\right) \phi_{(2)}^{T} O_{j}^{c r y}\right]\right\}\right\} & i=1 \ldots 24, j=1 \ldots 24 \\
\phi_{(2)}-\phi_{(1)}=(\triangle \phi-I) \phi_{(1)} & \text { orientation difference } \\
g_{i j, k}=\frac{\phi_{(2) i j}-\phi_{(1) i j}}{d_{k}} & \begin{array}{c}
\text { orientation gradient } \\
\text { (spacing d from EBSD scan) }
\end{array}
\end{array}
$$




\section{From local misorientations to GNDs}

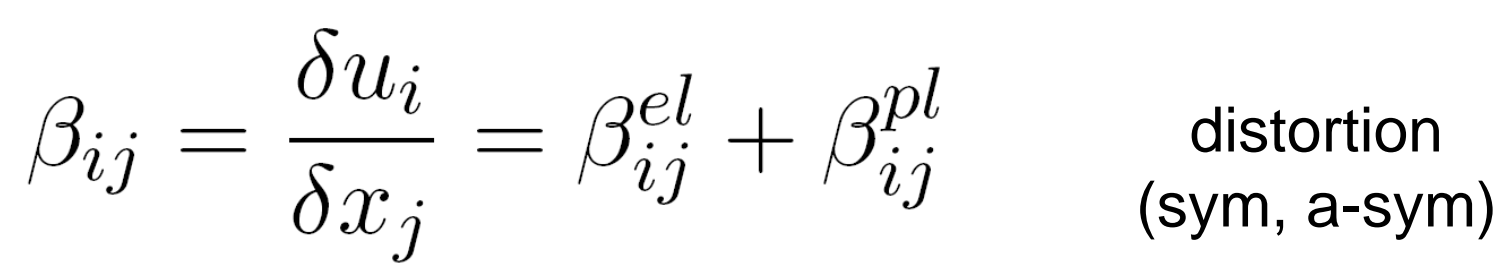

$\underline{u}=u(x, y, z)$
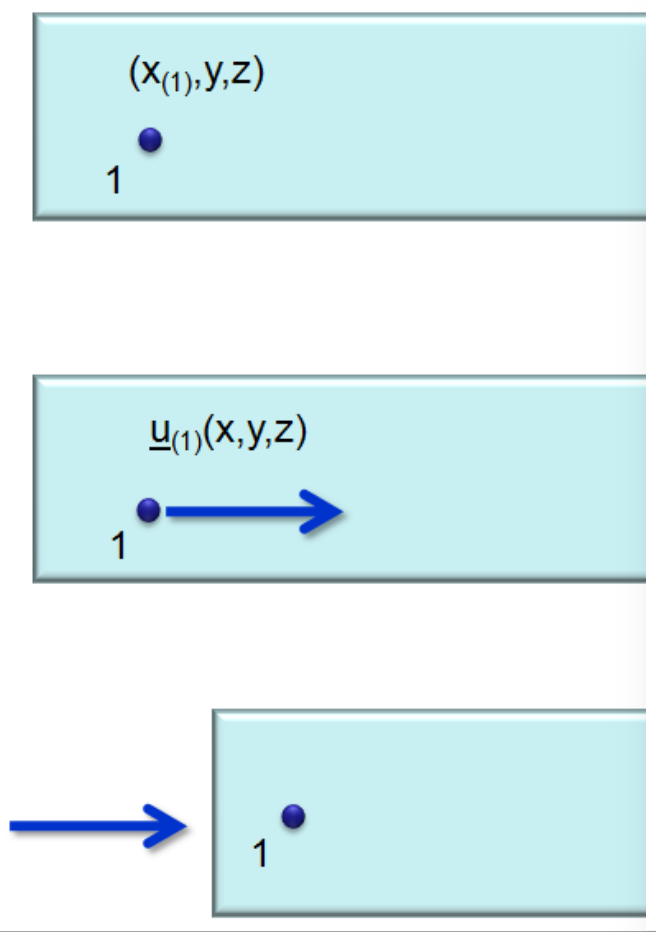

$\underline{u}=u(x, y, z)$

$\underline{\mathrm{u}}_{(1)}(\mathrm{x}, \mathrm{y}, \mathrm{z}) \neq \underline{\mathrm{u}}_{(2)}(\mathrm{x}, \mathrm{y}, \mathrm{z})$
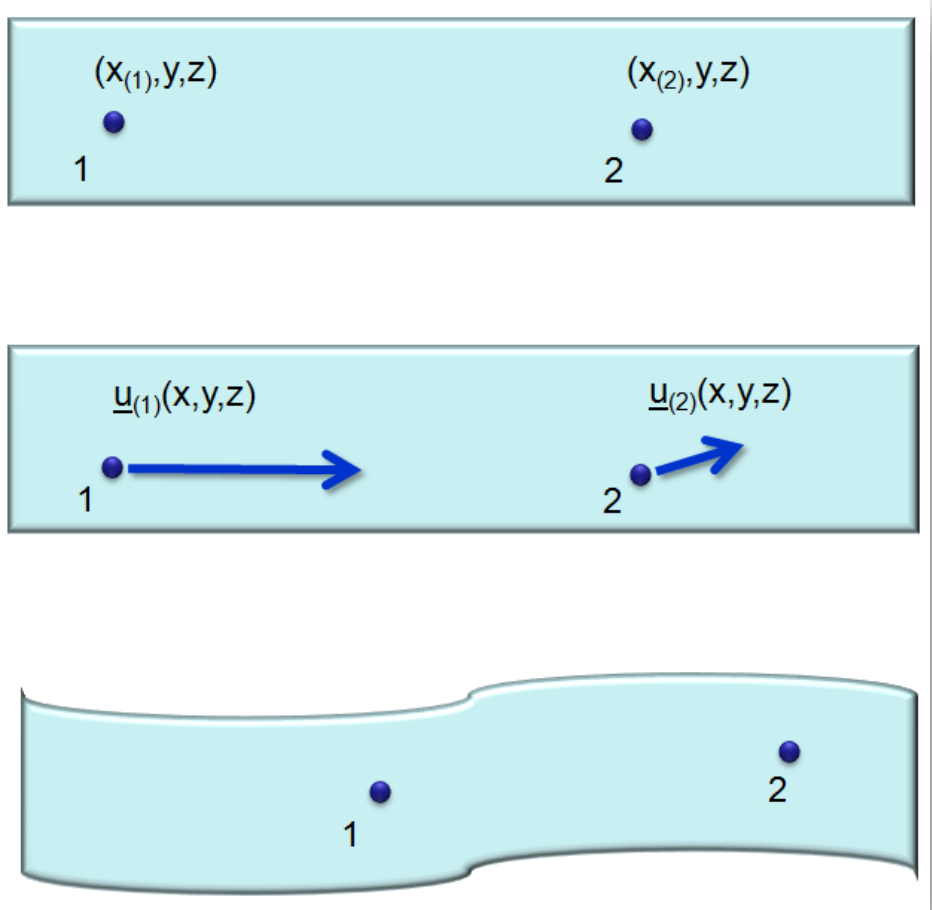


\section{From local misorientations to GNDs}

$$
\beta_{i j}=\frac{\delta u_{i}}{\delta x_{j}}=\beta_{i j}^{e l}+\beta_{i j}^{p l}
$$

(sym, a-sym)

$$
\begin{aligned}
& \alpha=\nabla \times \beta^{e l} \\
& \alpha_{p i}=e_{p k j}\left(\epsilon_{i j, k}^{e l}+g_{i j, k}\right) \\
& \alpha_{p i}=e_{p k j} g_{i j, k}
\end{aligned}
$$




\section{From local misorientations to GNDs}

Slip and line directions of dislocations for GNDs in a FCC crystal

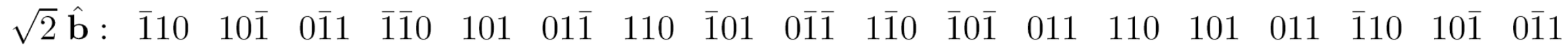
$\begin{array}{lllllllllllllllllll}\sqrt{6} \hat{\mathbf{t}}: & \overline{1} \overline{1} 2 & \overline{1} 2 \overline{1} & 2 \overline{1} \overline{1} & \overline{1} 1 \overline{2} & \overline{1} \overline{2} 1 & 211 & 1 \overline{1} \overline{2} & 121 & \overline{2} \overline{1} 1 & 112 & 1 \overline{2} \overline{1} & \overline{2} 1 \overline{1} & 110 & 101 & 011 & \overline{1} 10 & 10 \overline{1} & 0 \overline{1} 1\end{array}$ $\mathbf{B}=\mathbf{b}(\hat{\mathbf{t}} \cdot \mathbf{r})=(\mathbf{b} \otimes \hat{\mathbf{t}}) \mathbf{r} \quad$ Frank loop thrc

$$
\begin{aligned}
& \alpha_{i j}=\sum_{a=1}^{18} \rho_{g n d}^{a} b_{i}^{a} t_{j}^{a} \quad 18 \mathrm{~b}, \mathrm{t} \text { combination } \\
& \alpha_{i j}=\sum_{a=1}^{9} \rho_{g n d}^{a} b_{i}^{a} t_{j}^{a} \quad \text { Sbombations }
\end{aligned}
$$

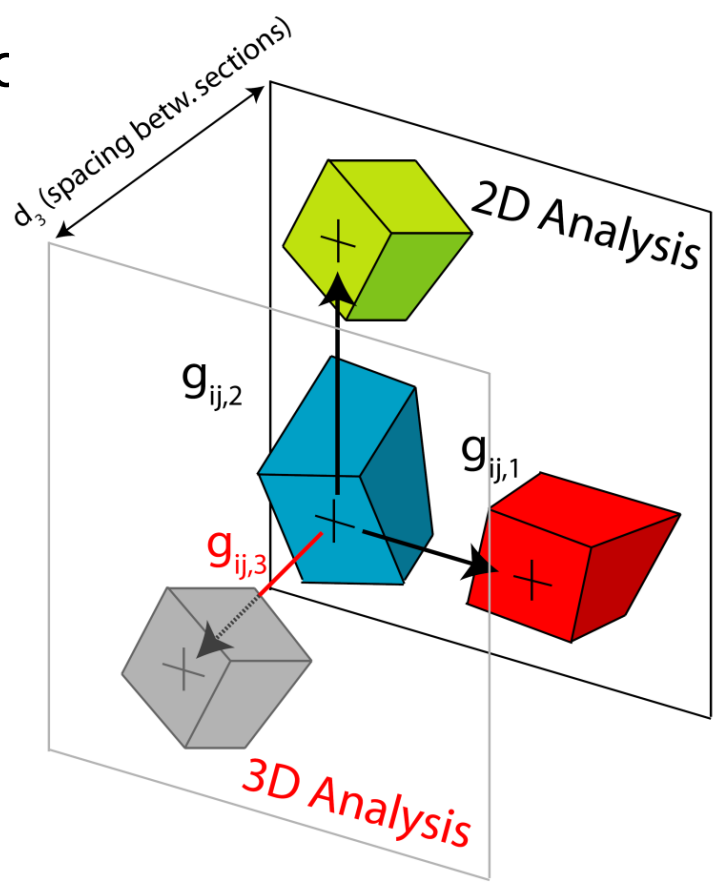




\section{D EBSD analysis of DP microstructure and texture}

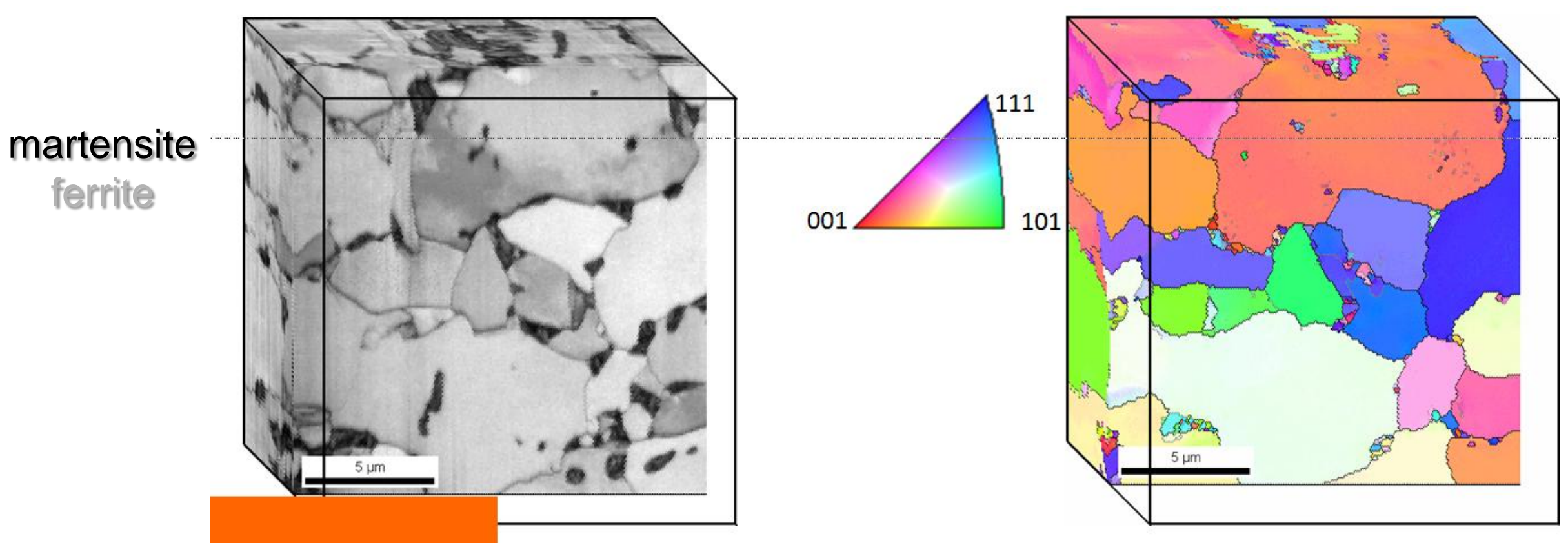


- Motivation

- Experiments

- Microstructure and texture evolution

- 3D tomographic analysis of interface regions

- Correlation to DP mechanical properties

- Ultra-fine grained DP

- Conclusions 


\section{D EBSD analysis of DP microstructure and texture}

Image Quality

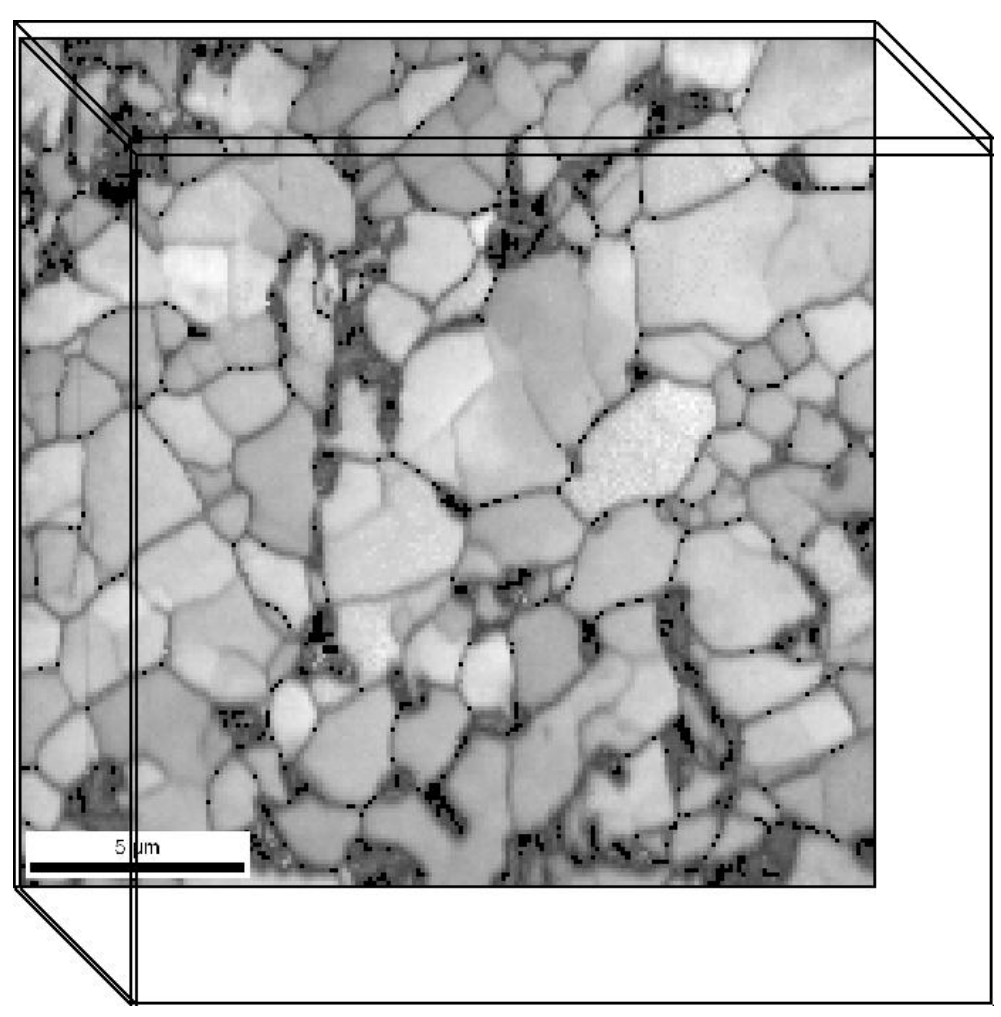

Kernel Average Misorientation (martensite highlighted in black)

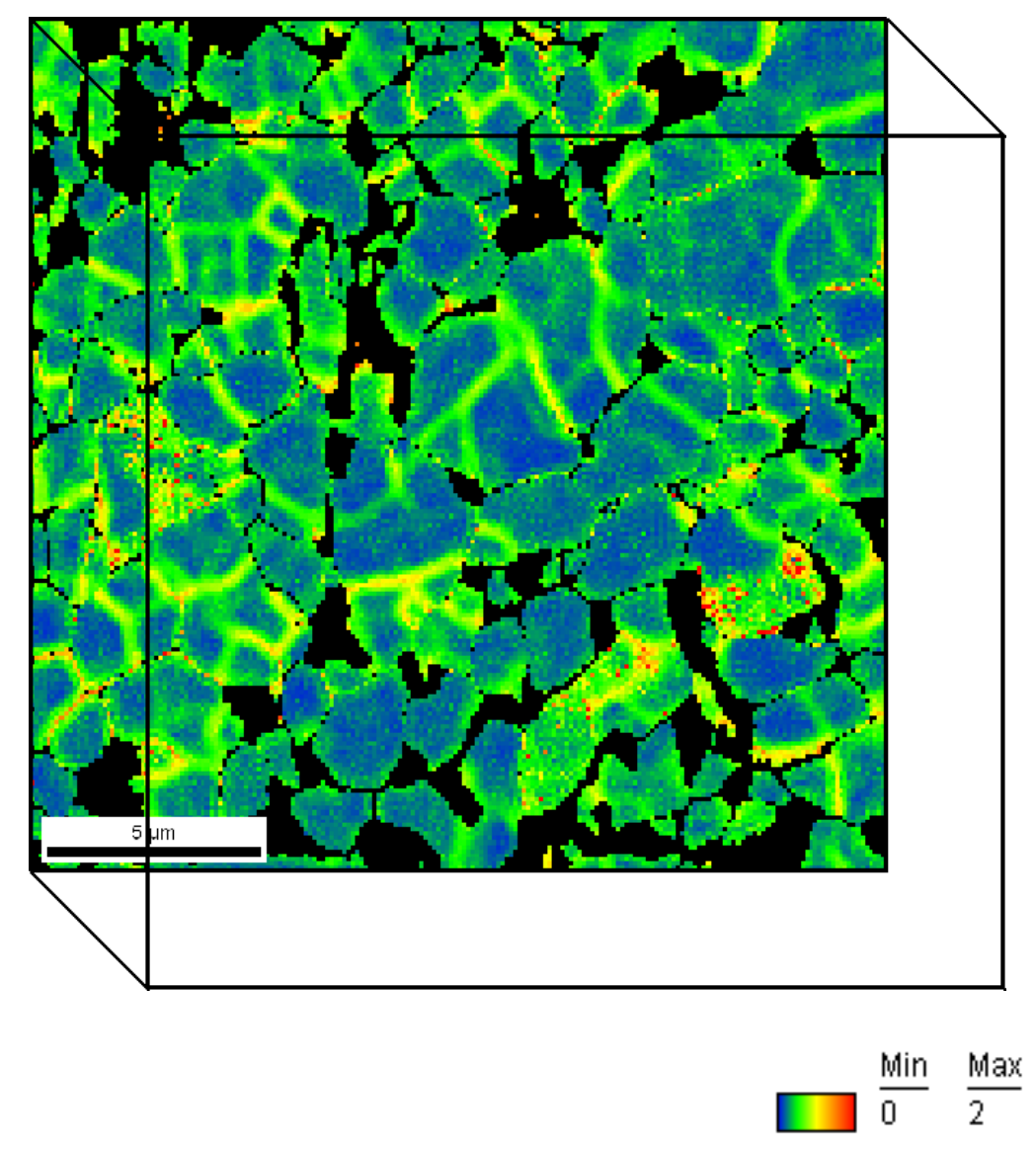




\section{KAM}

neighbor
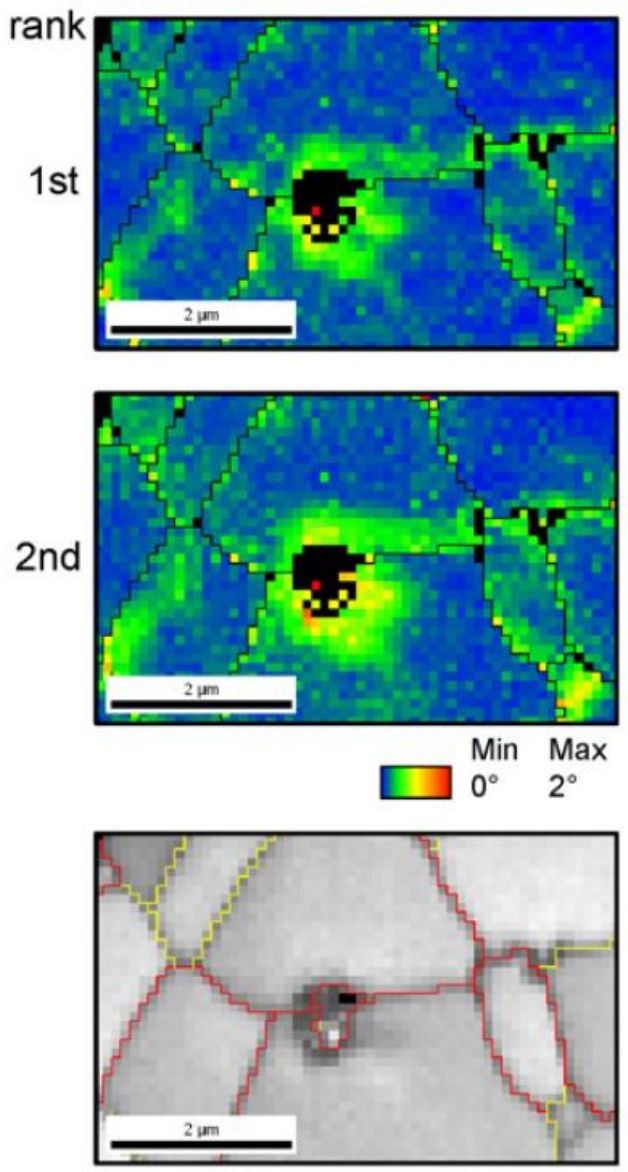

\section{GND density}
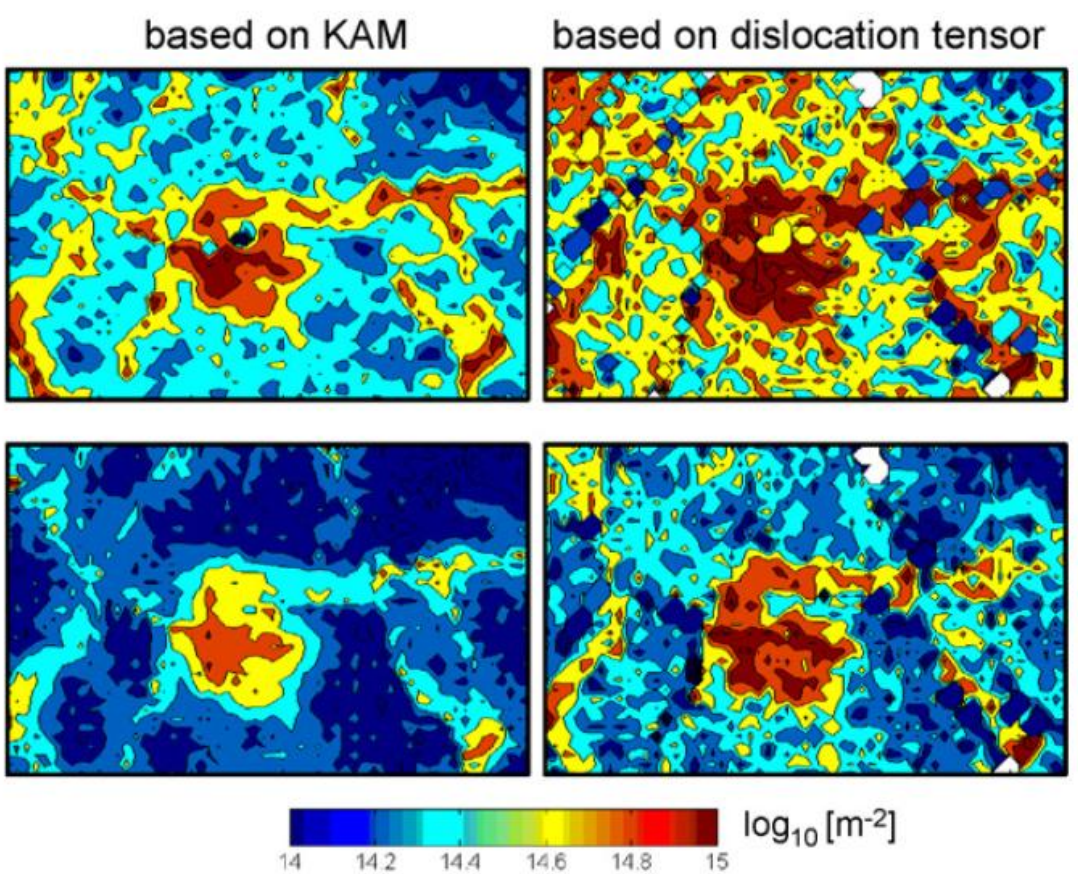

Image Quality

(grey scale)

grain

boundaries

Min Max

$2^{\circ} 15^{\circ}$

$-15^{\circ} 65^{\circ}$ 


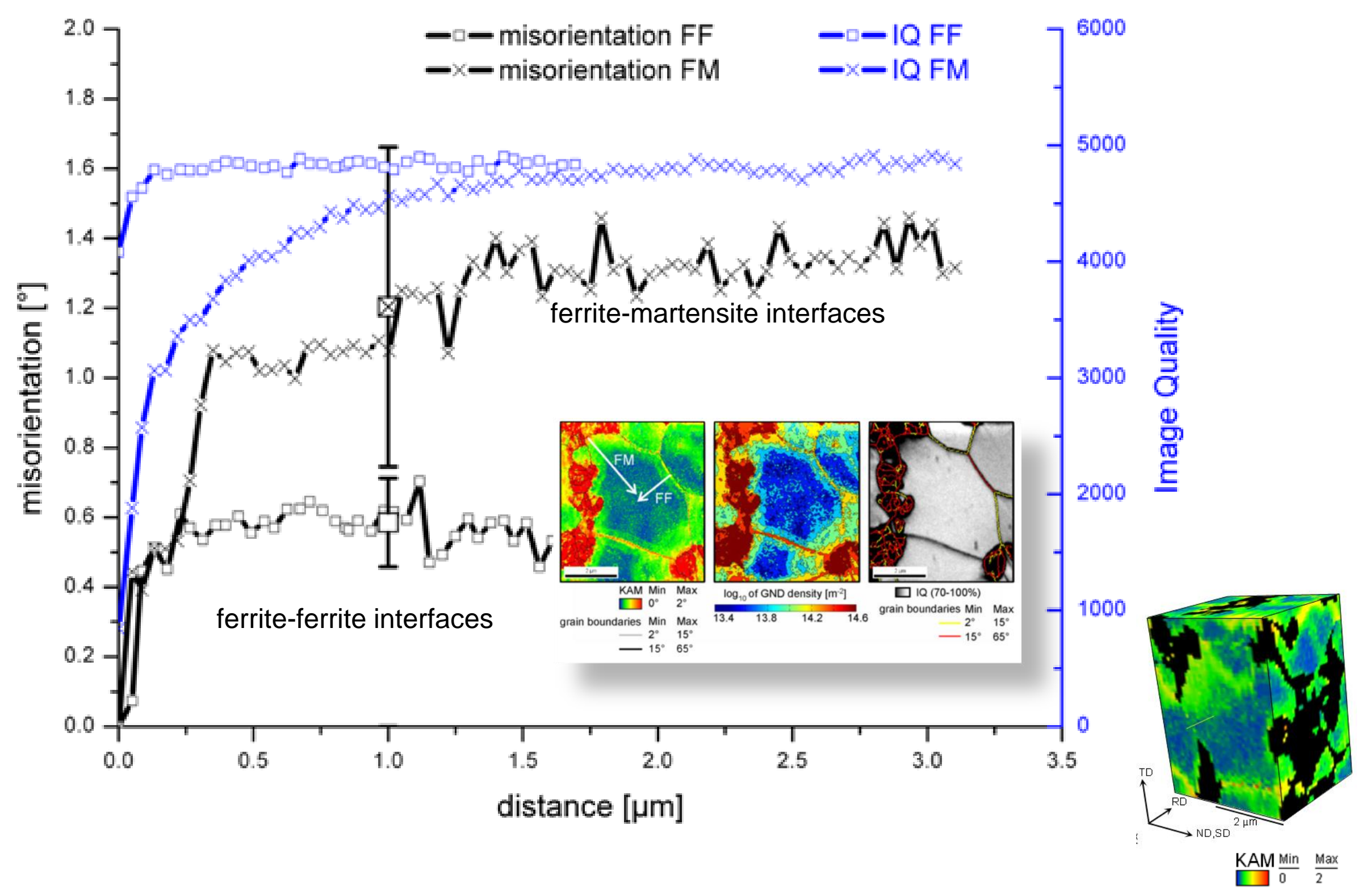


- Motivation

- Experiments

- Microstructure and texture evolution

- 3D tomographic analysis of interface regions

- Correlation to DP mechanical properties

- Ultra-fine grained DP

- Conclusions 


\section{Ultrafine grained DP steels}

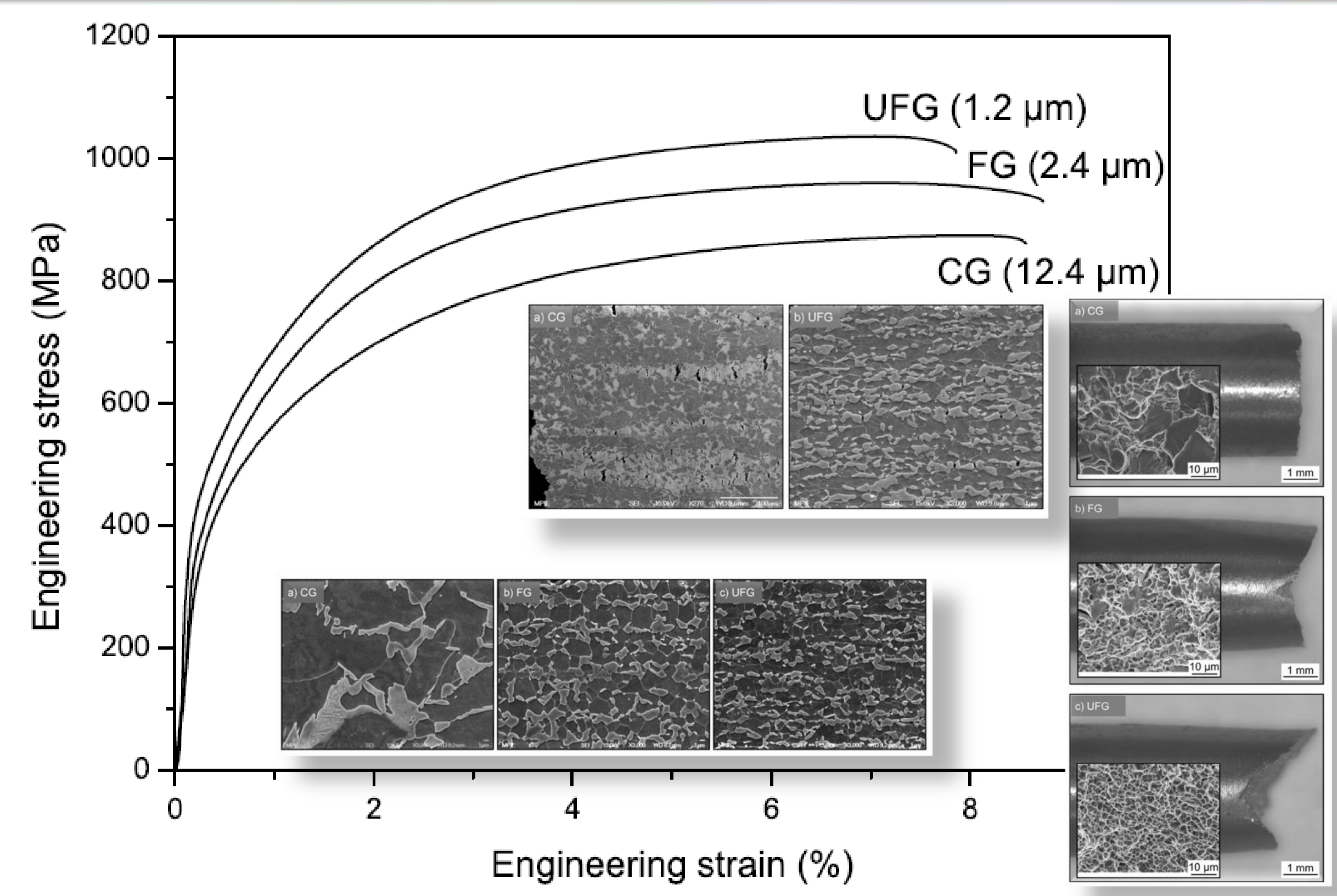




\section{D GND analysis of DP microstructure}

Coarse grained DP $(12.4 \mu \mathrm{m})$

Berkovich $50 \mathrm{~nm}$ Constant load $500 \mu \mathrm{N}$
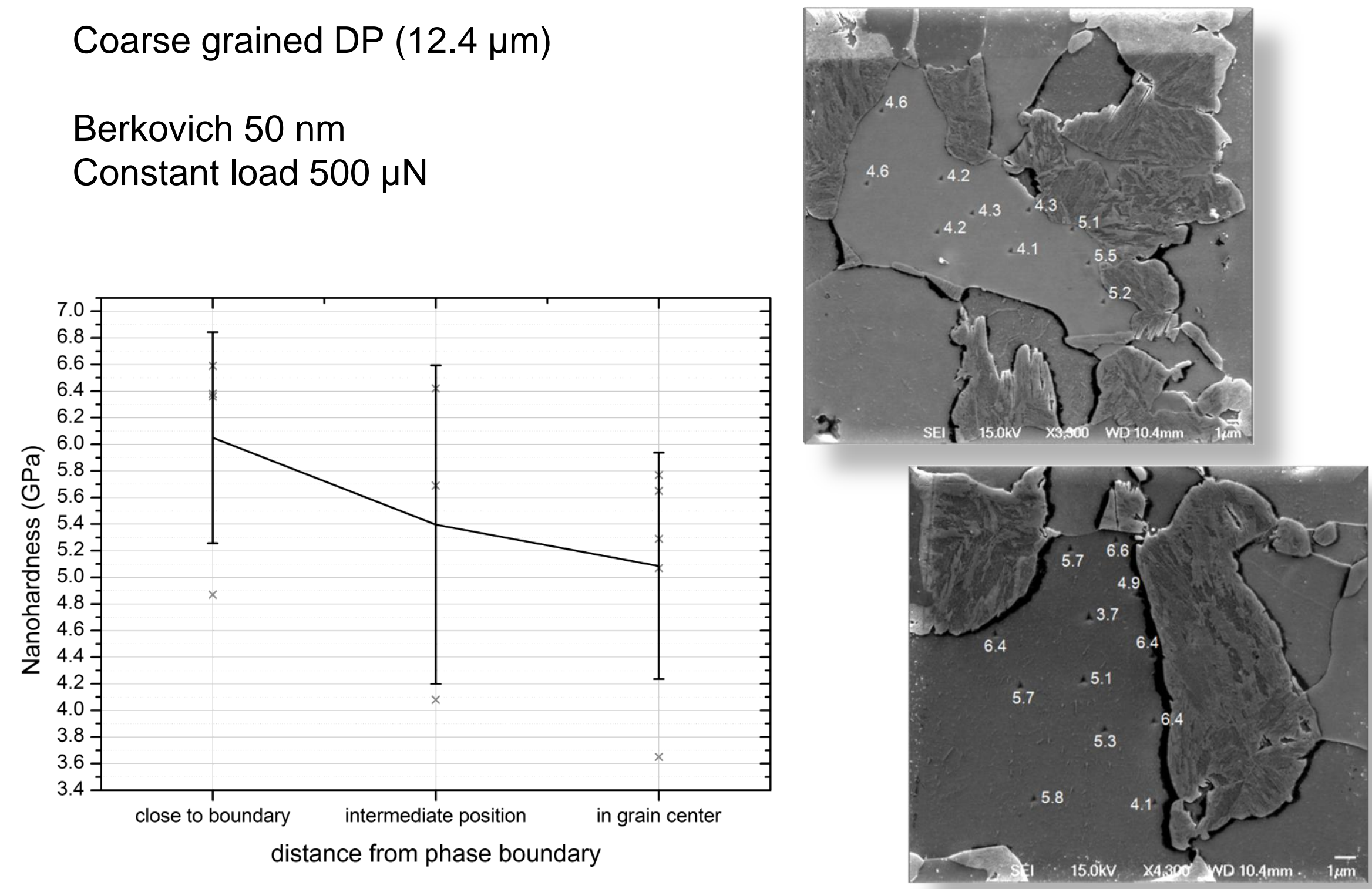
- Motivation

- Experiments

- Microstructure and texture evolution

- 3D tomographic analysis of interface regions

- Correlation to DP mechanical properties

- Ultra-fine grained DP

- Conclusions 


\section{Conclusions}

- Strong through-thickness gradients inherited from hot rolling

- Competition between RX and PT depends strongly on heat treatment conditions

- 3D tomographic analysis of texture and micromechanics

- Correlation of microstructure, texture, orientation gradients and interface strenght 


\section{The Dïsseldorf Hax-Planck Team}

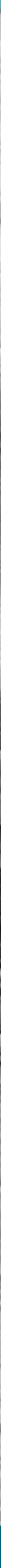




\section{Effect on microstructure}

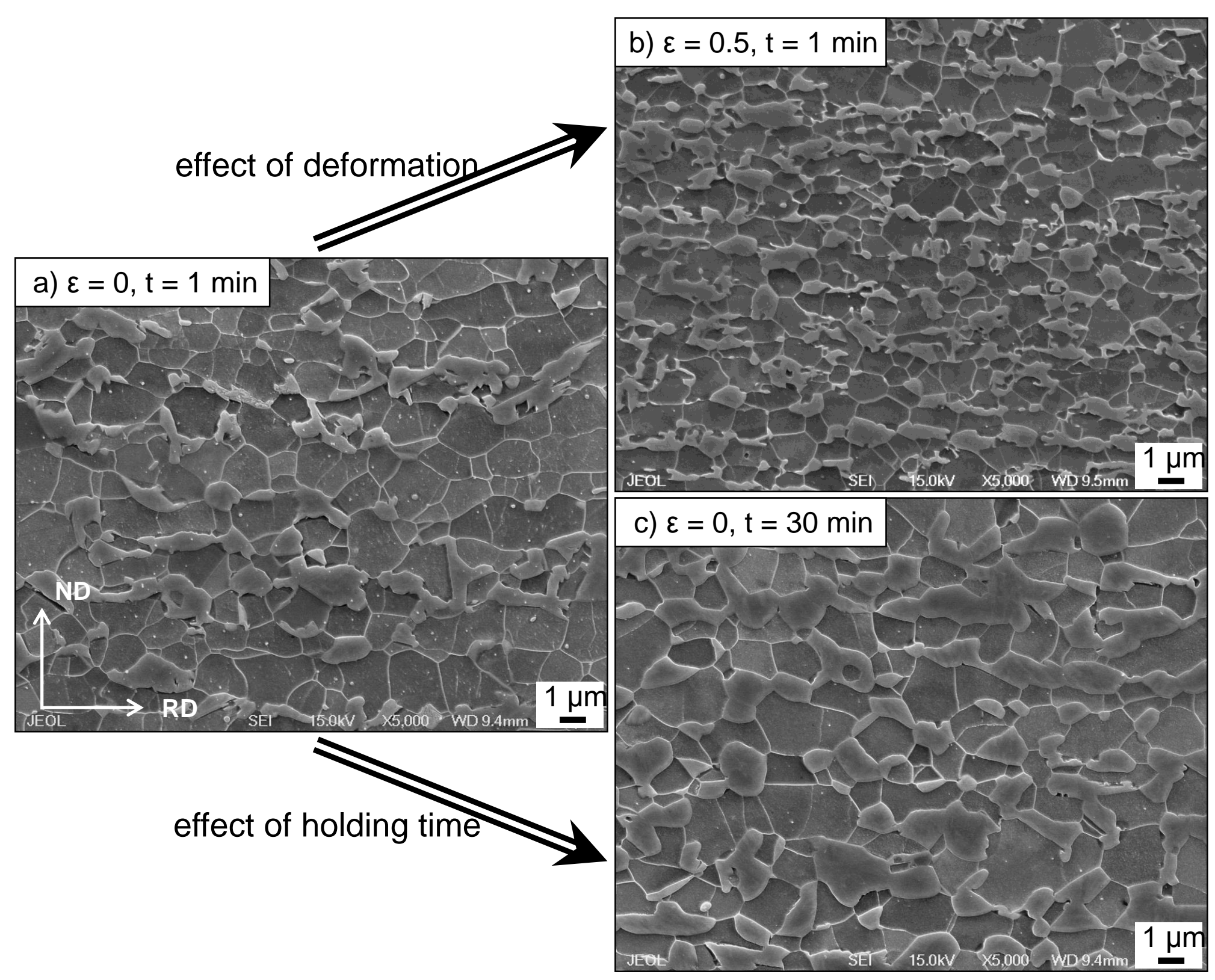




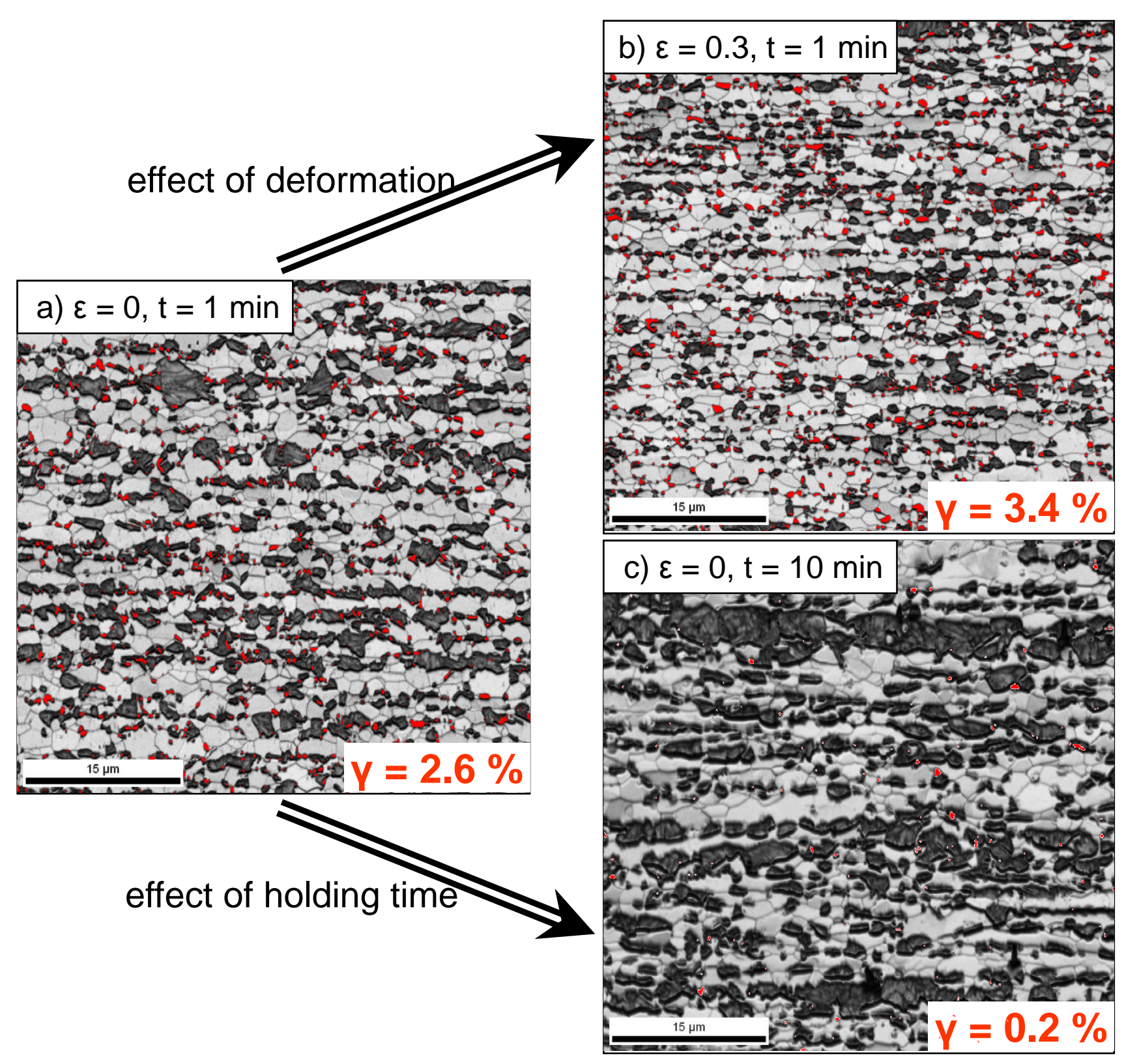




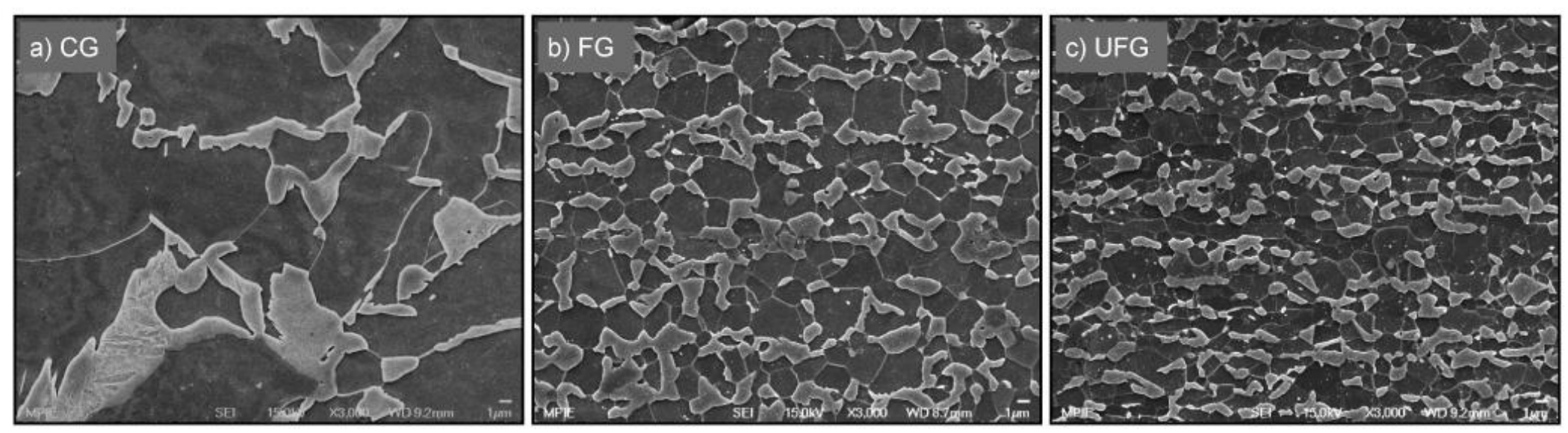

Figure 2: Microstructures used to evaluate the effect of grain refinement on mechanical properites. The a) coarse grained $(\mathrm{CG}), \mathrm{b})$ fine grained $(\mathrm{FG})$ and c) ultrafine grained (UFG) material were produced by the processing routes illustrated in Fig. 1 plus intercritical annealing for $3 \mathrm{~min}$ at $730{ }^{\circ} \mathrm{C}$ in a salt bath, followed by water quenching. 

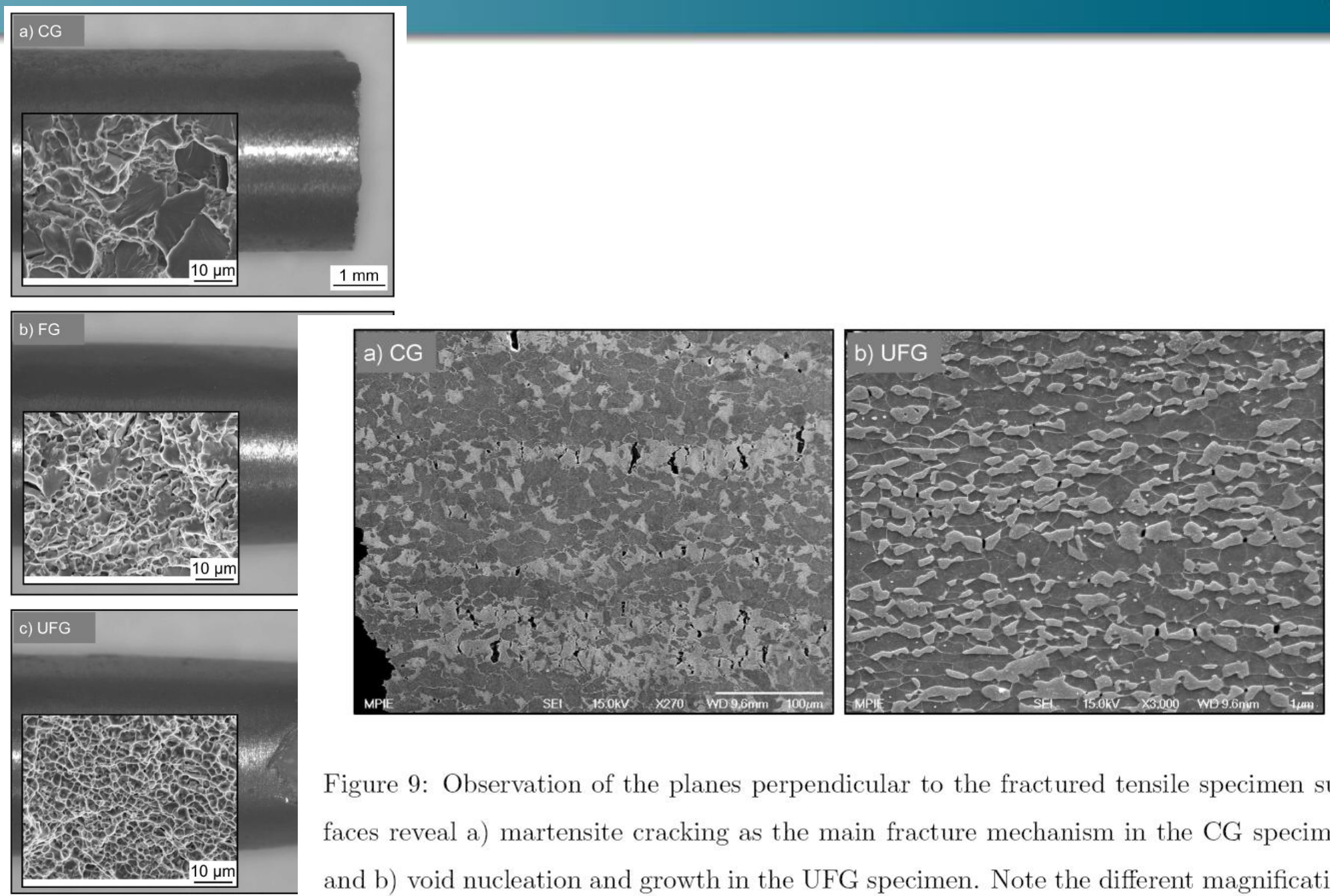

Figure 9: Observation of the planes perpendicular to the fractured tensile specimen surfaces reveal a) martensite cracking as the main fracture mechanism in the CG specimen and b) void nucleation and growth in the UFG specimen. Note the different magnification of the images. The tensile direction is horizontal. 


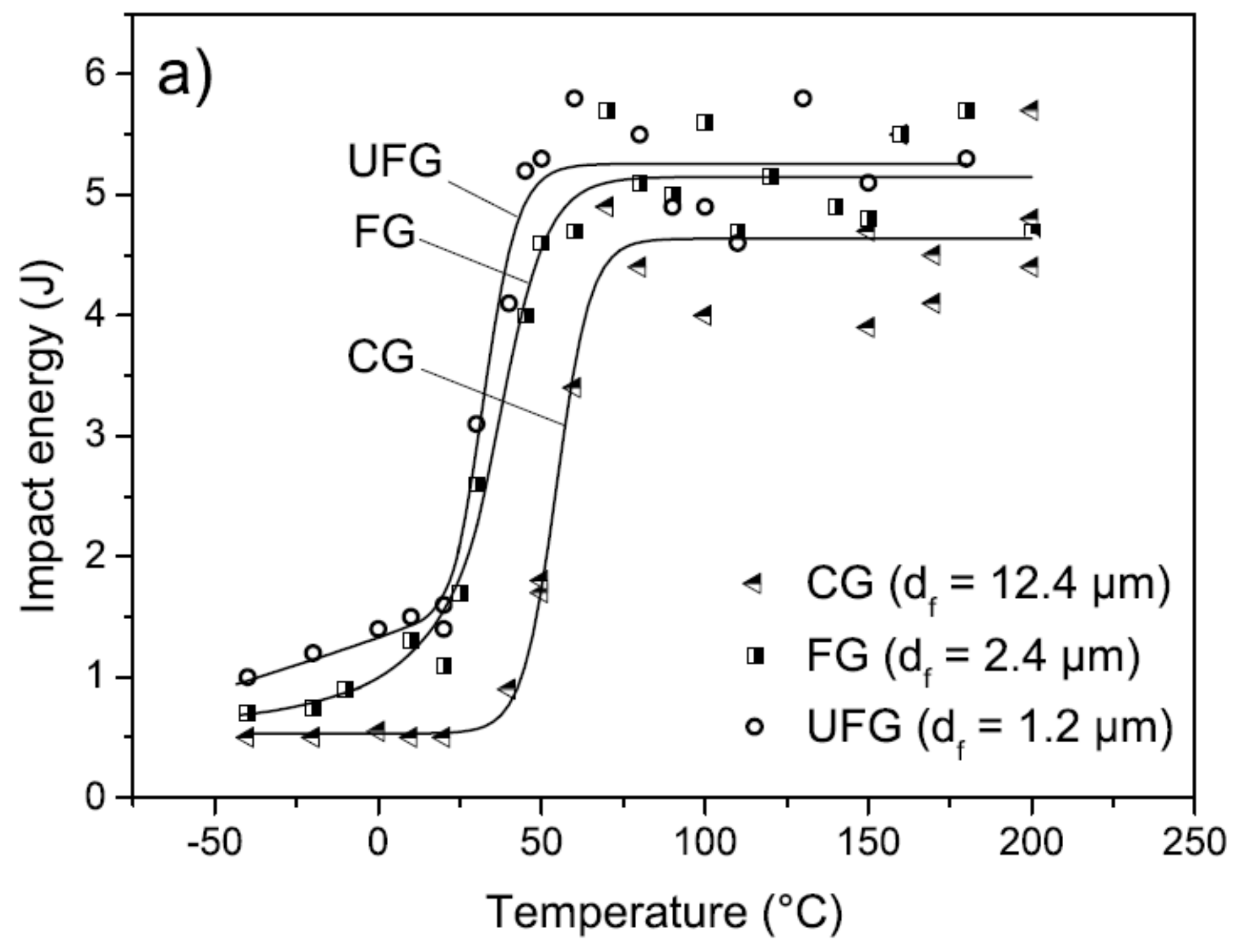

Max-Planck-Institut für Eisenforschung, Düsseldorf, Germany 


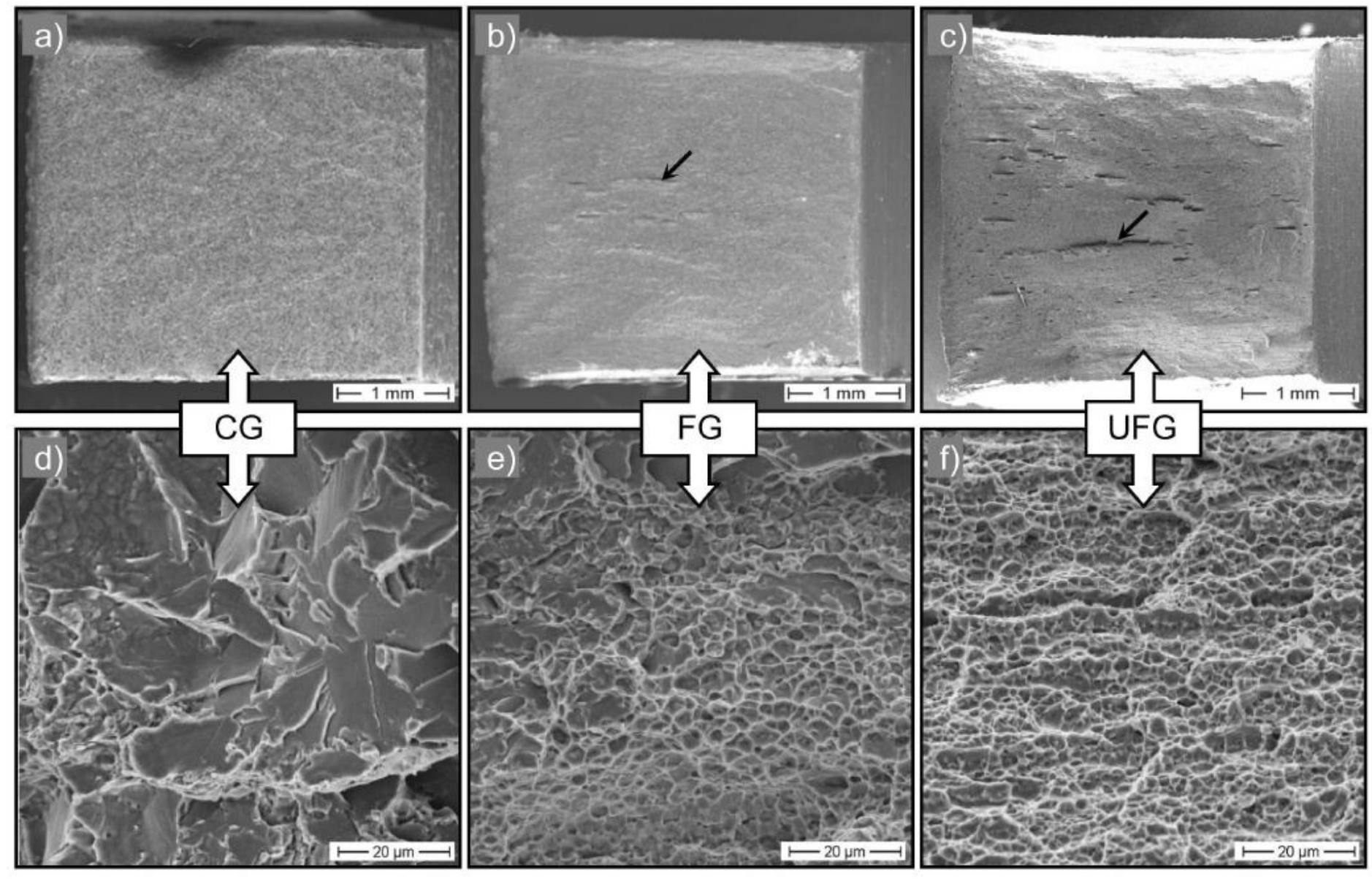

Figure 11: Fracture surfaces of subsize Charpy impact specimen fractured at room temperature. Like in the tensile specimen, grain refinement promotes ductile failure. Some delamination occurs in the FG and the UFG specimen (arrows). Rolling direction is Max-Pla horizontal. 